J. S. Okon and L. J. Ratliff, Jr.

Nagoya Math. J.

Vol. 122 (1991), 43-62

\title{
ON THE GRADE AND COGRADE OF A NOETHERIAN FILTRATION
}

\author{
J.S. OKON AND L.J. RATLIFF, Jr.
}

\section{§1. Introduction}

All rings in this paper are assumed to be commutative with identity and the terminology is standard.

Filtrations are a useful generalization of the sets of powers of an ideal $I$ in a ring $R$, and there are many important filtrations that are generally not such powers of an ideal. (For example: $\left\{Q^{(n)}\right\}_{n \geq 0}$, where $Q$ is a primary ideal; $\left\{\left(I^{n}\right)_{a}\right\}_{n \geq 0}$, where $\left(I^{n}\right)_{a}$ is the integral closure in $R$ of $I^{n}$; and, $\left\{u^{n} \mathscr{B} \cap R\right\}_{n \geq 0}$, where $\mathscr{B}$ is a graded subring of $R[u, t]$ that contains $R[u, t I]$ and $I$ is a given ideal of $R$.) They have played an important role in many research papers, and there are many results concerning them in the literature.

In several recent papers a number of important theorems concerning ideals in a Noetherian ring have been extended to Noetherian filtrations (e.g., see $[1,13,14,15,25,28,29])$. And in $[6,26]$ a number of results concerning the asymptotic prime divisors of an ideal are extended to finite collections of ideals. The results in this paper combine both types of extensions; specifically, we extend the "asymptotic" definitions (for an ideal) to a collection of $g \geq 1$ Noetheian filtrations. (As in the ideal case, it turns our that when working with filtrations $\phi_{1}, \cdots, \phi_{g}$ (with $g>1$ ), for the asymptotic prime divisor case it must be assumed that each $\phi_{i}(1)$ has height at least one, and for the essential prime divisor case the corresponding assumption is that each $\phi_{i}(1)$ is regular.) Our results then imply, as a special case, that the corresponding results hold for finite collections of ideals.

To be more specific, in this paper we extend the definitions of four types of prime divisors (viz, asymptotic, essential, quintasymptotic, and quintessential) from a single ideal to a collection $\Phi=\left(\phi_{1}, \cdots, \phi_{g}\right)$ of $g \geq 1$

Received December 28, 1989.

Revised September 7, 1990. 
Noetherian filtrations $\phi_{i}$. We show that each of these four types of prime divisors is, in fact, the corresponding type of prime divisor of certain of the ideals $\phi_{1}\left(n_{1}\right) \cdots \phi_{g}\left(n_{g}\right)$, so it follows that the known results for ideals concerning these types of prime divisors have a valid analog for $\Phi$. As an application of this, we introduce the four corresponding types of sequences in (and, over) $\Phi$ and the four corresponding grades and cogrades of $\Phi$. It is then shown that these are equal to the corresponding sequences, grades, and cogrades of $\phi_{1}\left(n_{1}\right) \cdots \phi_{g}\left(n_{g}\right)$, so the known results for ideals concerning these sequences, grades, and cogrades also have valid analogs for $\Phi$. As a final application of these results, it is shown that if $R$ is a quasi-unmixed local ring, then a $(\Phi)+\operatorname{acogd}(\Phi)=$ altitude $(R)$, where a denotes analytic spread and acogd denotes asymptotic cograde.

In Section 2 we define the four types of prime divisors for $\Phi$ and prove a few of their basic properties. In Section 3 we define the four types of sequences associated with $\Phi$ for these four types of prime divisors and use them to define the corresponding four types of grade and cograde of $\Phi$. Finally, in Section 4 we extend Rees' theorem concerning analytic spread and asymptotic cograde in quasi-unmixed local rings from ideals to finite collections of Noetherian filtrations.

\section{§ 2. The asymptotic prime divisors of a Noetherian filtration}

In this section we define four types of prime divisors associated with a finite collection of Noetherian filtrations and prove a few of their basic properties. For this, we begin with some definitions and notational conventions concerning filtrations.

(2.1) Definition. If $R$ is ring, then:

(2.1.1) A filtration $\phi=\{\phi(n)\}_{n \geq 0}$ on $R$ is a descending sequence of ideals $\phi(n)$ of $R$ such that $\phi(0)=R$ and $\phi(i) \phi(j) \subseteq \phi(i+j)$ for all nonnegative integers $i$ and $j$.

(2.1.2) If $k$ is a nonnegative integer, then $\phi^{(k)}$ denotes the subfiltration $\phi^{(k)}=\{\phi(n k)\}_{n \geq 0}$ of $\phi . \quad\left(\phi^{(0)}\right.$ is the filtration each of whose components is $R$.)

(2.1.3) If $\phi$ and $\gamma$ are filtrations on $R$, then $\phi=\gamma$ in case $\phi(n)=\gamma(n)$ for all $n \geq 0$, and $\phi \leq \gamma$ in case $\phi(n) \subseteq \gamma(n)$ for all $n \geq 0$.

(2.1.4) If $\phi$ and $\gamma$ are filtrations on $R$, then their sum $\phi+\gamma$ and prod$u c t \phi \gamma$ are defined to be the sequences of ideals $\phi+\gamma=\left\{\sum_{i=0}^{n} \phi(i) \gamma(n-i)\right\}_{n \geq 0}$ 
and $\phi \gamma=\{\phi(n) \gamma(n)\}_{n \geq 0}$. (It is readily checked that $\phi+\gamma$ and $\phi \gamma$ are filtrations on $R$ and that $\phi+\gamma$ is the smallest filtration $\theta$ on $R$ such that $\theta \geq \phi$ and $\theta \geq \gamma$, so it follows that if $\phi_{1}, \cdots, \phi_{g}$ are filtrations on $R$, then so are $\phi_{1}+\cdots+\phi_{g}$ and $\phi_{1} \cdots \phi_{g}$ and $\phi_{1}+\cdots+\phi_{g}$ is the smallest filtration $\theta$ on $R$ such that $\theta \geq \phi_{i}$ for $i=1, \cdots$, g.)

(2.1.5) The integral closure $\phi_{a}$ of a filtration $\phi$ on $R$ is the sequence of ideals $\phi_{a}=\left\{(\phi(n))_{a}\right\}_{n \geq 0}$, where $(\phi(n))_{a}$ is the integral closure of $\phi(n)$; therefore $(\phi(n))_{a}=\left\{x \in R ; x\right.$ satisfies an equation of the form $x^{k}+b_{1} x^{k-1}$ $+\cdots+b_{k}=0$, where $b_{i} \in(\phi(n))^{i}$ for $\left.i=1, \cdots, k\right\}$ (see (2.2.2)).

(2.1.6) The weak integral closure $\phi_{w}$ of a filtration $\phi$ on $R$ is the sequence of ideals $\phi_{w}=\left\{(\phi(n))_{w}\right\}_{n \geq 0}$, where $(\phi(n))_{w}$ is the weak integral closure of $\phi(n)$; therefore $(\phi(n))_{w}=\{x \in R ; x$ satisfies an equation of the form $x^{k}+b_{1} x^{k-1}+\cdots+b_{k}=0$, where $b_{i} \in \phi(n i)$ for $\left.i=1, \cdots, k\right\}$ (see (2.2.2)).

(2.1.7) If $\Phi=\left(\Phi_{1}, \cdots, \phi_{g}\right)$ is a collection of $g \geq 1$ filtrations on $R$, then the Rees ring $\mathscr{R}(R, \Phi)$ of $R$ with respect to $\Phi$ is the graded subring $\mathscr{R}(R, \Phi)=R\left[u_{1}, \cdots, u_{g},\left\{t_{1}^{i} \phi_{1}(i)\right\}_{i=1}^{\infty}, \cdots,\left\{t_{g}^{i} \phi_{g}(i)\right\}_{i=1}^{\infty}\right]$ of $R\left[u_{1}, \cdots, u_{g}, t_{1}, \cdots, t_{g}\right]$, where $t_{1}, \cdots, t_{g}$ are algebraically independent over $R$ and $u_{i}=1 / t_{i}$ for $i=1, \cdots, g$. If $\Phi=(\phi)$, then we will use $\mathscr{R}(R, \phi)$ in place of $\mathscr{R}(R, \Phi)$, and if $\phi=\left\{I^{n}\right\}_{n \geq 0}$ is the sequence of powers of an ideal $I$, then we will use $\mathscr{R}(R, I)$ in place of $\mathscr{R}(R, \phi)$.

(2.1.8) $\phi$ is said to be Noetherian in case $\mathscr{R}(R, \phi)$ is a Noetherian ring (see (2.1.7)). (It should be noted that this implies that $R$ is Noetherian, since it implies that $\mathscr{R}(R, \phi)[1 / u]=R[u, t]$ is Noetherian.) If $\phi$ is Noetherian, then we let $\operatorname{Int}(\phi)=\{e ; e$ is a positive integer such that $\phi(n+e)$ $=\phi(e) \phi(n)$ for all $n \geq e\}$. (By (2.2.3), $\operatorname{Int}(\phi) \neq \varnothing$ (when $\phi$ is Noetherian).) And if $\Phi=\left(\phi_{1}, \cdots, \phi_{g}\right)$ is a collection of $g$ Noetherian filtrations on $R$, then we let $\operatorname{Int}(\Phi)=\left\{\left(e_{1}, \cdots, e_{g}\right) ; e_{i} \in \operatorname{Int}(\phi)\right\}$.

(2.1.9) $\mathscr{P}_{g}$ (resp., $\mathscr{N}_{g}$ ) is the set of all $g$-tuples of positive (resp., nonnegative) integers. If $n=\left(n_{1}, \cdots, n_{g}\right) \in \mathscr{N}_{g}$, then $n(i)$ denotes $n_{i}$, the $i$-th component of $\mathfrak{n}$, and it will be said that $\mathfrak{n}$ is large in case each $\mathfrak{n}(i)$ is large. And if $m$ and $\mathfrak{n}$ are in $\mathscr{N}_{g}$ and $h$ is a positive integer, then $\mathfrak{m}+\mathfrak{n}, \mathfrak{m}-\mathfrak{n}, \mathfrak{m} \mathfrak{n}$, and $h \mathfrak{n}$ are defined in the usual componentwise manner, but we will only use $\mathfrak{m}-\mathfrak{n}$ when $\mathfrak{m} \geq \mathfrak{n}$ (that is, $\mathfrak{m}(i) \geq \mathfrak{n}(i)$ for $i=1$, $\cdots, g)$.

(2.1.10) If $\mathfrak{b}=\left(b_{1}, \cdots, b_{g}\right)$ (resp. $\mathfrak{S}=\left(I_{1}, \cdots, I_{g}\right)$ ) is a collection of $g$ (not necessarily distinct) elements (resp., ideals) of a ring related to $R$ 
and $\mathfrak{m} \in \mathscr{N}_{g}$, then $\mathfrak{b}^{\mathrm{m}}$ denotes the element $b_{1}^{\mathrm{m}(1)} \cdots b_{g}^{\mathrm{m}(g)}$, and $\mathfrak{I}^{\mathrm{m}}$ denotes the ideal $I_{1}^{\mathrm{m}(1)} \cdots I_{g}^{\mathrm{m}(g)}$.

(2.1.11) If $\Phi\left(\phi_{1}, \cdots, \phi_{g}\right)$ is a collection of $g$ (not necessarily distinct) filtrations on a ring $R$ and if $\mathfrak{m} \in \mathscr{N}_{g}$, then by $\Phi^{(\mathrm{m})}$ we mean the filtration $\phi_{1}^{(\mathfrak{m}(1))} \cdots \phi_{g}^{(\mathfrak{m}(g))}=\left\{\phi_{1}(n \mathfrak{m}(1)) \cdots \phi_{g}(n \mathfrak{m}(g))\right\}_{n \geq 0}$ (see (2.1.2) and (2.1.4)). Also, if $\mathfrak{n} \in \mathscr{N}_{g}$, then $\Phi^{(\mathrm{m})}(\mathfrak{n})$ denotes the ideal $\phi_{1}^{(\mathrm{m}(1))}(\mathfrak{n}(1)) \cdots \phi_{g}^{(\mathrm{m}(g))}(\mathfrak{n}(g))=$ $\phi_{1}(\mathfrak{m}(1) \mathfrak{n}(1)) \cdots \phi_{g}(\mathfrak{m}(g) \mathfrak{n}(g))$, and if $n$ is a positive integer, then $\Phi^{(\mathfrak{m})}(n)=$ $\left(\phi_{1}^{(\mathfrak{m}(1))} \cdots \phi_{g}^{(\mathfrak{m}(g))}\right)(n)=\phi_{1}^{(\mathfrak{m}(1))}(n) \cdots \phi_{g}^{(\mathfrak{m}(g))}(n)$. (It should be noted that: (a) for all $\mathfrak{m}, \mathfrak{n} \in \mathscr{N}_{g}, \Phi^{(\mathfrak{m})}(\mathfrak{n})=\Phi^{(\mathfrak{m n})}(1)=\Phi^{(\mathfrak{m n})}(1)$, where $1=(1, \cdots, 1) \in \mathscr{P}_{g} ;(\mathrm{b})$ for all $\mathfrak{m} \in \mathscr{N}_{g}, \Phi^{(\mathfrak{m})}(1)=\Phi^{(\mathfrak{m})}(1)=\Phi^{(1)}(\mathfrak{m})=\phi_{1}(\mathfrak{m}(1)) \cdots \phi_{g}(\mathfrak{m}(g))$; and, (c) if $\mathfrak{m}$, $\mathfrak{n} \in \mathscr{N}_{g}$ and either $\mathfrak{m}(i)=0$ or $\mathfrak{n}(i)=0$ for some $i=1, \cdots, g$, then $\Phi^{(\mathfrak{m n})}(1)$ $=\Phi^{(\mathfrak{m})}(\mathfrak{n})=\phi_{1}(\mathfrak{n}(1) \mathfrak{m}(1)) \cdots \phi_{i-1}(\mathfrak{n}(i-1) \mathfrak{m}(i-1)) R \phi_{i+1}(\mathfrak{n}(i+1) \mathfrak{m}(i+1)) \cdots$ $\phi_{g}(\mathfrak{n}(g) \mathfrak{m}(g))$.

Most of the definitions in (2.1) have previously appeared in the literature. However, the weak integral closure $\phi_{w}$ of $\phi$ is called the integral closure of $\phi$ in [28]. This seems like appropriate terminology to us, but there is already quite a lot of literature where $\phi_{w}$ is called the weak integral closure of $\phi$ and the filtration $\phi_{a}$ is called the integral closure of $\phi$, so we decided to stay with the older terminology.

(2.2) lists four facts concerning these definitions that will be needed in what follows.

(2.2) Remark. (2.2.1) Note that if $\phi$ is a filtration on a ring $R$, then the weak integral closure $(\phi(n))_{w}$ of $\phi(n)=\phi^{(n)}(1)$ is completely determined by the subfiltration $\phi^{(n)}$ of $\phi$, since $\phi^{(n)}(i)=\phi(n i)$ for all $i \geq 0$. Extending this, if $\Phi=\left(\phi_{1}, \cdots, \phi_{g}\right)$ is a collection of finitely many filtrations on $R$ and $\mathfrak{m}, \mathfrak{n} \in \mathscr{N}_{g}$, then we use $\left(\Phi^{(\mathfrak{m})}(\mathfrak{n})\right)_{w}$ to denote the weak integral closure of the ideal $\Phi^{(\mathfrak{m})}(\mathfrak{n})=\Phi^{(1)}(\mathfrak{m n})=\Phi^{(\mathfrak{m n})}(1)$ with respect to the filtration $\Phi^{(\mathrm{mn})}=\left\{\phi_{1}(\mathrm{im}(1) \mathfrak{n}(1)) \cdots \phi_{g}(i \mathfrak{m}(g) \mathfrak{n}(g))\right\}_{i \geq 0}$.

(2.2.2) If $\phi$ is a filtration on $R$, then it is shown in [13, (4.2.1) and (2.2)] that $\phi_{a}$ and $\phi_{w}$ are filtrations on $R$ such that $\phi \leq \phi_{a} \leq \phi_{w}$. Also, if $\phi$ is Noetherian and $e \in \operatorname{Int}(\phi)$ (see (2.1.8)), then $\phi(i n e)=(\phi(n e))^{i}$ for all positive integers $i$ and $n$, so it follows from (2.1.5) and (2.1.6) that $(\phi(n e))_{a}$ $=(\phi(n e))_{w}$ for all $n \geq 1$. Further, if $\Phi=\left(\phi_{1}, \cdots, \phi_{g}\right)$ is a collection of $g$ Noetherian filtrations on $R$ and $e_{i} \in \operatorname{Int}\left(\phi_{i}\right)$ for $i=1, \cdots, g$, and if $\mathrm{e}=$ $\left(e_{i}, \cdots, e_{g}\right)$, then a short computation shows that $\Phi^{(1)}(\mathrm{ine})=\left(\phi_{1}(\mathfrak{n}(1) e(1))^{i(1)}\right.$ $\cdots\left(\phi_{g}(\mathfrak{n}(g) e(g))\right)^{i(g)}$ for all $i, n \in \mathscr{N}_{g}$, so in particular it follows from (2.1.5), 
(2.1.6), and (2.2.1) that $\left(\Phi^{(1)}(\mathfrak{n} e)\right)_{a}=\left(\Phi^{(1)}(\mathfrak{n} e)\right)_{w}$ for all $\mathfrak{n} \in \mathscr{N}_{g}$.

(2.2.3) If $R$ is Noetherian, then it is shown in [1, (2.2) and (3.6)] together with $[16,(2.7)]$ that the following are equivalent: $\phi$ is Noetherian; there exists a positive integer $e$ such that $\phi(n+e)=\phi(e) \phi(n)$ for all $n \geq e$; and, $\mathscr{R}(R, \phi)$ is finitely generated over $R$.

(2.2.4) If $R$ is Noetherian and $\Phi=\left(\phi_{1}, \cdots, \phi_{g}\right)$ is a finite collection of Noetherian filtrations on $R$, then it is shown in [16, (3.1)] that $\phi_{1}+\cdots$ $+\phi_{g}$ and $\phi_{1} \cdots \phi_{g}$ are Noetherian. And it is shown in [16, (4.2)] that if $\phi$ is Noetherian, then so is $\phi^{(m)}$ (see (2.1.2)) for all $m \geq 0$ (the assumption that $R$ is an analytically unramified semi-local ring in [16] is not used to prove this part of the result), so it follows that $\Phi^{(\mathrm{m})}$ is Noetherian for all $\mathfrak{m} \in \mathscr{N}_{g}$.

In (2.3) we define the various prime divisors of a Noetherian filtration and of a finite collection of Noetherian filtrations. Three of these definitions are new (for filtrations) to this paper, but (2.3.2) was given and studied in $[13,14,28]$. However, the definitions are stated in a manner to emphasize the analogs between quintasymptotic-asymptotic prime divisors and quintessential-essential prime divisors, so (2.3.2) differs from the definition given in $[13,14,28]$. (But it is shown in (2.6) that the definitions are equivalent.) Also, when these four definitions are compared, it will be noted that (2.3.2) is somewhat different from the other three, since the designator $\hat{A}^{*}$ for the asymptotic prime divisors of an ideal is replaced with $\mathscr{A}_{w}$ for filtrations, while the designators $\hat{\mathscr{Q}}^{*}, \mathscr{Q}$, and $\mathscr{E}$ for the quintasymptotic, the quintessential, and the essential prime divisors of an ideal are continued for filtrations. The reason for this is because $\left(u^{n} \mathscr{R}(R, I)\right)_{a} \cap R=\left(I^{n}\right)_{a}$ and $\left(u^{n} \mathscr{R}(R, \phi)\right)_{a} \cap R=(\phi(n))_{w}$ (which may properly contain $\left.(\phi(n))_{n}\right)$, and it is noted in (2.5.3) (resp., (2.6.1)) that $\hat{A}^{*}(I)=\cup\left\{\operatorname{Ass}\left(R /\left(I^{n}\right)_{a} ; n \geq 1\right\} \quad\left(\operatorname{resp} . \mathscr{A}_{w}(\phi)=\bigcup\left\{\operatorname{Ass}\left(R /(\phi(n))_{w}\right) ; n \geq 1\right\}\right)\right.$, so $\mathscr{A}_{w}(\phi)$ is the appropriate filtration analog of $\hat{A}^{*}(I)$.)

(2.3) Definition. Assume that $R$ is Noetherian, let $I$ be an ideal in $R$, let $\phi$ be a Noetherian filtration on $R$, and let $\Phi=\left(\phi_{1}, \cdots, \phi_{g}\right)$ be a collection of $g$ Noetherian filtrations on $R$. Then:

(2.3.1) $\hat{\mathscr{Q}}^{*}(I)=\{P \in \operatorname{Spec}(R) ; I \subseteq P$ and there exists a minimal prime divisor $z$ of zero in the completion $\left(R_{P}\right)^{*}$ of $R_{P}$ such that $I\left(R_{P}\right)^{*}+z$ is $P\left(R_{P}\right)^{*}$-primary\}, $\hat{\mathscr{Q}}^{*}(\phi)=\hat{\mathscr{Q}}^{*}(\phi(1))$, and $\hat{\mathscr{Q}}^{*}(\Phi)=\hat{\mathscr{Q}}^{*}\left(\Phi^{(1)}(1)\right)$ (see $(2.1 .11)(\mathrm{b})$ ). The members of $\hat{\mathscr{Q}}^{*}(I)$ (resp., $\hat{\mathscr{Q}}^{*}(\phi), \hat{\mathscr{Q}}^{*}(\Phi)$ ) are called the quintasymptotic 
prime divisors of $I$ (resp., $\phi, \Phi)$.

(2.3.2) $\hat{A}^{*}(I)=\left\{p \cap R ; p \in \hat{\mathscr{Q}}^{*}(u \mathscr{R}(R, I))\right\}$ (see (2.3.1) and (2.1.7)), $\mathscr{A}_{w}(\phi)$ $=\left\{p \cap R ; p \in \widehat{\mathscr{Q}}^{*}(u \mathscr{R}(R, \phi))\right\}$, and $\mathscr{A}_{w}(\Phi)=\left\{p \cap R ; p \in \widehat{\mathscr{Q}}^{*}(\mathfrak{H} \mathscr{R}(R, \Phi))\right\}$, where $\mathfrak{u}=\left(u_{1}, \cdots, u_{g}\right)$ (see $\left.(2.1 .10)\right)$. The members of $\hat{A}^{*}(I)$ (resp., $\left.\mathscr{A}_{w}(\phi), \mathscr{A}_{w}(\Phi)\right)$ are called the asymptotic prime divisors of $I$ (resp., $\phi, \Phi)$.

(2.3.3) $\mathscr{2}(I)=\{P \in \operatorname{Spec}(R) ; I \subseteq P$ and there exists a (not necessarily minimal) prime divisor $z$ of zero in the completion $\left(R_{P}\right)^{*}$ of $R_{P}$ such that $I\left(R_{P}\right)^{*}+z$ is $P\left(R_{P}\right)^{*}$-primary\}, $\mathscr{2}(\phi)=\mathscr{2}(\phi(1))$, and $\mathscr{Q}(\Phi)=\mathscr{2}\left(\Phi^{(1)}(1)\right)$. The members of $\mathscr{2}(I)$ (resp., $2(\phi), \mathscr{Q}(\Phi)$ ) are called the quintessential prime divisors of $I$ (resp., $\phi, \Phi)$.

(2.3.4) $\mathscr{E}(I)=\{p \cap R ; p \in \mathscr{Q}(u \mathscr{R}(R, I))\}, \mathscr{E}(\phi)=\{p \cap R ; p \in \mathscr{Q}(u \mathscr{R}(R, \phi))\}$, and $\mathscr{E}(\Phi)=\left\{p \cap R ; p \in \mathscr{Q}\left(\mathfrak{u}^{1} \mathscr{R}(R, \Phi)\right)\right\}$, where $\mathfrak{u}=\left(u_{1}, \cdots, u_{g}\right)$. The members of $\mathscr{E}(I)$ (resp., $\mathscr{E}(\Phi), \mathscr{E}(\Phi)$ ) are called the essential prime divisors of $I$ (resp., $\phi, \Phi)$.

(It should be noted that the notation and terminology conerning these prime divisors for ideals was changed in [5]; in papers before [5], what we termed "quintessential" in (2.3.3) was called "essential" (and $E$ was used in place of 2 ), and what we termed "essential" in (2.3.4) was called "Uf-essential" (and $U$ was used in place of $\mathscr{E}$ ). (Some reasons for these changes are given in the appendix in [5].) The reader should keep these changes in mind when checking references.)

The sets $\mathscr{A}_{w}(\phi)$ and $\mathscr{A}_{w}(\Phi)$ were defined differently in $[13,14,28]$, so we show in (2.6) that these different definitions are equivalent. Before this, however, we first show that the definitions of $\hat{\mathscr{Q}}^{*}(\Phi)$ and $\mathscr{Q}(\Phi)$ imply that these sets are the sets of the corresponding type of prime divisor for each of the ideals $\Phi(\mathfrak{n})$ for all $\mathfrak{n} \in \mathscr{P}_{g}$.

(2.4) Theorem. Let $R$ be a Noetherian ring and let $\Phi=\left(\phi_{1}, \cdots, \phi_{g}\right)$ be a collection of $g \geq 1$ Noetherian filtrations on $R$. Then:

(2.4.1) $\hat{\mathscr{Q}}^{*}(\Phi)=\hat{\mathscr{Q}}^{*}\left(\Phi^{(1)}(\mathfrak{n})\right)$ for all $\mathfrak{n} \in \mathscr{P}_{g}$.

(2.4.2) $\mathscr{Q}(\Phi)=\mathscr{Q}\left(\Phi^{(1)}(\mathfrak{n})\right)$ for all $\mathfrak{n} \in \mathscr{P}_{g}$.

Proof. It follows from the definition of a filtration $\phi$ that $\operatorname{Rad}(\phi(1))$ $=\operatorname{Rad}(\phi(n))$ for all $n \geq 1$, so it follows from (2.1.11) (and since $\operatorname{Rad}\left(\phi_{i}(n)\right.$ ) $=\operatorname{Rad}\left(\phi_{i}(m)\right)$ for $i=1, \cdots, g$ and for all positive integers $n$ and $m$ ) that $\operatorname{Rad}\left(\Phi^{(1)}(\mathfrak{n})\right)=\operatorname{Rad}\left(\Phi^{(1)}(1)\right)$ for all $\mathfrak{n} \in \mathscr{P}_{g}$. Also, if $I$ and $J$ are ideals with the same radical, then it follows from (2.3.1) (resp., (2.3.3)) that $\hat{\mathscr{Q}}^{*}(I)=$ $\widehat{\mathscr{Q}}^{*}(J)$ (resp., $\mathscr{Q}(I)=2(J)$ ). (2.4.1) (resp., (2.4.2)) readily follows from this 
together with (2.3.1) (resp., (2.3.3)).

q.e.d.

To prove some additional results concerning these types of prime divisors of $\Phi$ we need to use some known results concerning such prime divisors in the ideal case, so we next list these.

(2.5) Remark. If $I$ is an ideal in a Noetherian ring $R$, then:

(2.5.1) It should be noted that if $\phi=\left\{I^{n}\right\}_{n \geq 0}$ is the filtration of powers of $I$, then $\phi(1)=I$ and $\mathscr{R}(R, \phi)=\mathscr{R}(R, I)$, so (2.3) shows that $\hat{\mathscr{Q}}^{*}(\phi)=$ $\hat{\mathscr{Q}}^{*}(I), \mathscr{A}_{w}(\phi)=\hat{A}^{*}(I), \mathscr{Q}(I)=\mathscr{Q}(I)$, and $\mathscr{E}(\phi)=\mathscr{E}(I)$.

(2.5.2) It is shown in [10, (2.2)] that if $I=b R$ is a regular principal ideal, then $\hat{\mathscr{Q}}^{*}(I)=\hat{A}^{*}(I)$.

(2.5.3) It is shown in [19, (2.4)] that if $P \in \operatorname{Ass}\left(R /\left(I^{n}\right)_{a}\right)$ for some $n \geq 1$, then $P \in \operatorname{Ass}\left(R /\left(I^{n+k}\right)_{a}\right)$ for all $k \geq 0$, and it is shown in [19, (2.7)] that $\hat{A}^{*}(I)=\operatorname{Ass}\left(R /\left(I^{n}\right)_{a}\right)$ for all large $n$.

(2.5.4) It is readily checked that if $b_{1}, \cdots, b_{g}$ are regular nonunits in $R$, then $\hat{A}^{*}\left(b_{1} \cdots b_{g} R\right)=\bigcup\left\{\hat{A}^{*}\left(b_{i} R\right) ; i=1, \cdots, g\right\}=\bigcup\left\{\operatorname{Ass}\left(R /\left(\mathfrak{b}^{\mathfrak{n}} R\right)_{a}\right) ; \mathfrak{n} \in \mathscr{N}_{g}\right\}$, where $\mathfrak{b}=\left(b_{1}, \cdots, b_{g}\right)$.

In (2.6) we show that the definitions of $\mathscr{A}_{w}(\phi)$ and $\mathscr{A}_{w}(\Phi)$ in (2.3.2) are equivalent to their definitions in $[13,14,28]$.

(2.6) Theorem. If $R$ is a Noetherian ring, then:

(2.6.1) If $\phi$ is a Noetherian filtration on $R$, then $\mathscr{A}_{w}(\phi)=\{P \in \operatorname{Spec}(R)$; $P \in \operatorname{Ass}\left(R /(\phi(n))_{w}\right)$ for some $\left.n \geq 1\right\}=\operatorname{Ass}\left(R /(\phi(n))_{w}\right)$ for all large $n$.

(2.6.2) If $\Phi=\left(\phi_{1}, \cdots, \phi_{g}\right)$ is a collection of $g$ Noetherian filtrations on $R$ such that height $\left(\phi_{i}(1)\right) \geq 1$ for $i=1, \cdots, g$, then $\mathscr{A}_{w}(\Phi)=\mathscr{A}_{w}\left(\phi_{1} \cdots \phi_{g}\right)=$ $\left\{P \in \operatorname{Spec}(R) ; P \in \operatorname{Ass}\left(R /\left(\Phi^{(1)}(\mathfrak{n})\right)_{w}\right)\right.$ for some nonzero $\left.\mathfrak{n} \in \mathscr{N}_{g}\right\}=\operatorname{Ass}\left(R /\left(\Phi^{(1)}(\mathfrak{n})\right)_{w}\right)$ for all large $\mathfrak{n} \in \mathscr{P}_{g}$.

Proof. For (2.6.1) let $S=\left\{P \in \operatorname{Spec}(R) ; P \in \operatorname{Ass}\left(R /(\phi(n))_{w}\right)\right.$ for some $n \geq 1\}$ and let $\mathscr{R}=\mathscr{R}(R, \phi)$. Then in [13, (3.1.2)] $\mathscr{A}_{w}(\phi)$ is defined to be the set $S$, and it is shown in [13, (3.3.2)] that $S=\left\{p \cap R ; p \in \hat{A}^{*}(u \mathscr{R})\right\}$. Also, $\hat{A}^{*}(u \mathscr{R})=\hat{\mathscr{Q}}^{*}(u \mathscr{R})$, by (2.5.2), so it follows from (2.3.2) that $\mathscr{A}_{w}(\phi)$ $=S$. Further, it is shown in $[13,(3.4 .3)]$ that $S=\operatorname{Ass}\left(R /(\phi(n))_{w}\right)$ for all large $n$, so (2.6.1) holds.

For (2.6.2) let $T=\left\{P \in \operatorname{Spec}(R) ; P \in \operatorname{Ass}\left(R /\left(\Phi^{(1)}(\mathfrak{n})\right)_{w}\right.\right.$ ) for some nonzero $\left.\mathfrak{n} \in \mathscr{N}_{g}\right\}$. Then since height $\left(\phi_{i}(1)\right) \geq 1$ for $i=1, \cdots, g$, it is shown in [14, (2.7.3)] that $T=\mathscr{A}_{w}\left(\phi_{1} \cdots \phi_{g}\right)=\operatorname{Ass}\left(R /\left(\Phi^{(1)}(\mathfrak{n})\right)_{w}\right)$ for all large $\mathfrak{n} \in \mathscr{P}_{g}$, so it remains to show that $\mathscr{A}_{w}(\Phi)=T$. 
For this, if $g=1$, then the conclusion holds by (2.6.1), so it may be assumed that $g>1$ and that the conclusion holds for $g-1$ filtrations. Let $\mathscr{R}=\mathscr{R}(R, \Phi)$, let $\mathfrak{u}=\left(u_{1}, \cdots, u_{g}\right)$, and let $P \in T$, say $P \in \operatorname{Ass}\left(R /\left(\Phi^{(1)}(\mathfrak{n})\right)_{w}\right)$. Then in $[14,(4.1 .2)]$ it is shown that $\left(\mathfrak{u}^{\mathfrak{n}} \mathscr{R}\right)_{a} \cap R=\left(\Phi^{(1)}(\mathfrak{n})\right)_{w}$ for all $\mathfrak{n} \in \mathscr{N}_{g}$, so there exists $p \in \operatorname{Ass}\left(\mathscr{R} /\left(\mathfrak{u}^{n} \mathscr{R}\right)_{a}\right)$ such that $p \cap R=P$. Also, $u_{1}, \cdots, u_{g}$ are regular elements in $\mathscr{R}$, so it follows from (2.5.4) that $\operatorname{Ass}\left(\mathscr{R} /\left(\mathfrak{u}^{\mathfrak{n}} \mathscr{R}\right)_{a}\right)$ $\subseteq \hat{A}^{*}\left(\mathfrak{u}^{1} \mathscr{R}\right)$, and (2.5.2) shows that $\hat{A}^{*}\left(\mathfrak{u}^{1} \mathscr{R}\right)=\hat{\mathscr{Q}}^{*}\left(\mathfrak{u}^{1} \mathscr{R}\right)$. Therefore $p \in$ $\hat{\mathscr{Q}}^{*}\left(\mathfrak{u}^{1} \mathscr{R}\right)$ and $P=p \cap R$, so it follows from (2.3.2) that $T \subseteq \mathscr{A}_{w}(\Phi)$.

For the opposite inclusion let $P \in \mathscr{A}_{w}(\Phi)$ and let $p \in \widehat{\mathscr{Q}}^{*}\left(\mathfrak{u}^{1} \mathscr{R}\right)$ such that $p \cap R=P$. To show that $P \in T$ we now consider the two cases: (a) $u_{i} \notin p$ for some $i=1, \cdots, g$; and, (b) $\left(u_{1}, \cdots, u_{g}\right) \mathscr{R} \subseteq p$. If (a) holds, then let $\mathscr{B}=R\left[u_{1}, \cdots, u_{i-1}, u_{i+1}, \cdots, u_{g},\left\{t_{1}^{j} \phi_{1}(j)\right\}_{j=1}^{\infty}, \cdots,\left\{t_{i-1}^{j} \phi_{i-1}(j)\right\}_{j=1}^{\infty},\left\{t_{i+1}^{j} \phi_{i+1}(j)\right\}_{j=1}^{\infty}\right.$, $\left.\cdots,\left\{t_{g}^{j} \phi_{g}(j)\right\}_{j=1}^{\infty}\right]$, so $\mathscr{B} \subseteq \mathscr{R} \subseteq \mathscr{B}\left[u_{i}, t_{i}\right]=\mathscr{R}\left[1 / u_{i}\right]$. Also, since $t_{i}$ is an indeterminate and $u_{i}=1 / t_{i}$, it follows from [10, (3.4) and (3.6)] that $q=$ $p \mathscr{R}\left[1 / u_{i}\right] \cap \mathscr{B} \in \widehat{\mathscr{Q}}^{*}\left(\left(u_{1} \cdots u_{i-1} u_{i+1} \cdots u_{g}\right) \mathscr{B}\right)$. Therefore by induction on $g \geq 1$ it follows that $p \cap R=q \cap R \in \operatorname{Ass}\left(R /\left(\Gamma^{(1)}(\mathfrak{n})\right)_{w}\right.$ ) for some nonzero $\mathfrak{n} \in \mathscr{N}_{g-1}$, where $\Gamma=\left(\phi_{1}, \cdots, \phi_{i-1}, \phi_{i+1}, \cdots, \phi_{g}\right)$. Thus it follows that $P=p \cap R \in$ $\operatorname{Ass}\left(R /\left(\Phi^{(1)}(\mathfrak{m})\right)_{w}\right)$, where $\mathfrak{m}$ is the element in $\mathscr{N}_{g}$ such that $\mathfrak{m}(j)=\mathfrak{n}(j)$ for $j=1, \cdots, i-1, \mathfrak{m}(i)=0$, and $\mathfrak{m}(j)=\mathfrak{n}(j-1)$ for $j=i+1, \cdots, g$, so $P \in T$, hence $\mathscr{A}_{w}(\Phi) \subseteq T$ when (a) holds.

If (b) holds, then since $p \in \widehat{\mathscr{Q}}^{*}\left(\mathfrak{u}^{1} \mathscr{R}\right)=\hat{A}^{*}\left(\mathfrak{u}^{1} \mathscr{R}\right)$ (by hypothesis and (2.5.2)), it follows from (2.5.3) that if $m$ is large and $\mathfrak{m}=(m, \cdots, m) \in \mathscr{P}_{g}$, then $p \in \operatorname{Ass}\left(\mathscr{R} /\left(\mathfrak{u}^{\mathrm{m}} \mathscr{R}\right)_{a}\right)$. Also, $\left(\mathfrak{u}^{\mathrm{m}} \mathscr{R}\right)_{a}$ is homogeneous, so it follows from [2, Proposition 1, p. 283] that there exists a homogeneous element $b t^{\mathfrak{n}} \in \mathscr{R}$ such that $p=\left(\mathfrak{u}^{\mathrm{m}} \mathscr{R}\right)_{a}: b \mathrm{t}^{\mathrm{n}} \mathscr{R}$. Now possibly $\mathfrak{n}(i)$ is negative for some $i=$ $1, \cdots, g$. If this is the case, then let $\mathscr{B}$ be as in the preceding paragraph, so $\mathscr{R}=\mathscr{B}\left[u_{i},\left\{t_{i}^{j} \phi_{i}(j)\right\}_{j=1}^{\infty}\right] \cong \mathscr{R}\left(\mathscr{B}, \phi_{i} \mathscr{B}\right)$. Also, $p \in \operatorname{Ass}\left(\mathscr{R} /\left(u_{i}^{m} \mathscr{R}\right)_{a}\right)$ for all large $m$ (since $u_{i} \in p$ ), so it is shown in the proof of [12, (3.6.2)] that $t_{i}^{j} \phi_{i}(j) \not \subset p$ for some $j \geq 1$. Thus there exists an element $c \in \phi_{i}(j)$ such that $c t_{i}^{j} \notin p$, so $p=\left(\left(\mathfrak{u}^{\mathrm{m}}\right) \mathscr{R}\right)_{a}: b t^{\mathrm{n}}\left(c t_{i}^{j}\right)^{h} \mathscr{R}$ for all $h \geq 1$. By repeating this for each $i=$ $1, \cdots, g$ such that $\mathfrak{n}(i)<0$ it may de assumed that $p=\left(\left(\mathfrak{u}^{\mathrm{m}}\right) \mathscr{R}\right)_{a}: d \mathrm{t}^{\mathrm{a}} \mathscr{R}$ for some nonzero $\mathfrak{q} \in \mathscr{N}_{g}$ and for some $d \in \Phi^{(1)}(\mathfrak{q})$. Now it is shown in [14, (4.1.2)] that $\left(\left(\mathfrak{u}^{\mathrm{m}}\right) \mathscr{R}\right)_{a}: d \mathfrak{t}^{\mathfrak{a}} \mathscr{R} \cap R=\left(\Phi^{(1)}(\mathfrak{m}+\mathfrak{q})\right)_{w}: d R$, so it follows that $p \cap R$ $=\left(\Phi^{(1)}(\mathfrak{m}+\mathfrak{q})\right)_{w}: d R$, hence $P=p \cap R \in \operatorname{Ass}\left(R /\left(\Phi^{(1)}(\mathfrak{n})_{w}\right)\right.$ for some $\mathfrak{n} \in \mathscr{P}_{g}$. Therefore $P \in T$, so $\mathscr{A}_{w}(\Phi) \subseteq T$ when (b) holds, so it follows that (2.6.2) holds.

q.e.d. 
The first part of the following theorem will help shorten several proofs below, and the second part shows that the asymptotic and essential prime divisors of a single filtration $\phi$ are, respectively, the asymptotic and essential prime divisors of the ideal $\phi(e)$ for each $e \in \operatorname{Int}(\phi)$.

(2.7) Theorem. If $R$ is a Noetherian ring, then:

(2.7.1) Let $\Phi=\left(\phi_{1}, \cdots, \phi_{g}\right)$ and $\Gamma=\left(\gamma_{1}, \cdots, \gamma_{g}\right)$ be collections of $g \geq 1$ Noetherian filtrations on $R$, let $\mathfrak{n} \in \mathscr{P}_{g}$, and assume that $\mathscr{R}=\mathscr{R}(R, \Phi)$ is an integral extension ring of $\mathscr{B}=R\left[u_{1}^{\mathrm{n}(1)}, \cdots, u_{g}^{\mathrm{n}(g)},\left\{t_{1}^{i \mathrm{n}(1)} \gamma_{1}(\mathrm{in}(1))\right\}_{i \geq 1}, \cdots\right.$, $\left.\left\{t_{g}^{i n(g)} \gamma_{g}(i n(g))\right\}_{i \geq 1}\right]$. Then $\mathscr{A}_{w}(\Phi)=\mathscr{A}_{w}\left(\Delta_{n}\right)$ and $\mathscr{E}(\Phi)=\mathscr{E}\left(\Delta_{n}\right)$, where $\Delta_{\mathfrak{n}}=$ $\left(\gamma_{1}^{(\mathfrak{n}(1))}, \cdots, \gamma_{g}^{(\mathrm{n}(g))}\right)$.

(2.7.2) If $\phi$ is a filtration on $R$, then $\mathscr{A}_{w}(\phi)=\hat{A}^{*}(\phi(e))$ and $\mathscr{E}(\phi)=$ $\mathscr{E}(\phi(e))$ for all $e \in \operatorname{Int}(\phi)$.

Proof. For (2.7.1) it follows from the structure of $\mathscr{R}$ and $\mathscr{B}$ that $z \cap \mathscr{B} \in \operatorname{Ass}(\mathscr{B})$ for all $z \in \operatorname{Ass}(\mathscr{R})$, so $\hat{A}^{*}\left(\mathfrak{u}^{\mathfrak{n}} \mathscr{B}\right)=\left\{p \cap \mathscr{B} ; p \in \hat{A}^{*}\left(\mathfrak{u}^{\mathrm{n}} \mathscr{R}\right)\right\}$ by [22, (3.3.4)]. Also, $\hat{A}^{*}\left(\mathfrak{u}^{\mathfrak{n}} \mathscr{R}\right)=\hat{A}^{*}\left(\mathfrak{u}^{1} \mathscr{R}\right)$, by [8, (2.4.3)]. Further, $\hat{A}^{*}\left(\mathfrak{u}^{1} \mathscr{R}\right)$ $=\hat{\mathscr{Q}}^{*}\left(\mathfrak{u}^{1} \mathscr{R}\right)$ and $\hat{A}^{*}\left(\mathfrak{u}^{\mathrm{n}} \mathscr{B}\right)=\hat{\mathscr{Q}}^{*}\left(\mathfrak{u}^{\mathfrak{n}} \mathscr{B}\right)$ by $(2.5 .2)$, and it is clear that $\mathscr{B} \cong$ $\mathscr{R}\left(R, \Delta_{\mathfrak{n}}\right)$, where $\Delta_{\mathfrak{n}}=\left(\gamma_{1}^{(\mathfrak{n}(1))}, \cdots, \gamma_{g}^{(\mathfrak{n}(g))}\right)$. Therefore it follows from (2.3.2) and the isomorphism that $\mathscr{A}_{w}(\Phi)=\mathscr{A}_{w}\left(\Delta_{\mathfrak{n}}\right)$. Moreover, $\mathscr{E}\left(\mathfrak{u}^{\mathrm{n}} \mathscr{B}\right)=\{p \cap \mathscr{B}$; $\left.p \in \mathscr{E}^{(}\left(\mathfrak{u}^{\mathrm{n}} \mathscr{R}\right)\right\}$, by $[4,(2.5 .4)]$, and $\mathscr{E}\left(\mathfrak{u}^{\mathrm{n}} \mathscr{R}\right)=\mathscr{E}^{\mathfrak{N}}\left(\mathfrak{u}^{1} \mathscr{R}\right)$, by [4, (2.5.6)], so it follows from (2.3.4) and the isomorphism that $\mathscr{E}(\Phi)=\mathscr{E}\left(\Delta_{\mathfrak{n}}\right)$.

For (2.7.2) let $e \in \operatorname{Int}(\phi)$, let $\mathscr{S}=\mathscr{R}(R, \phi)$, and let $\mathscr{C}=R\left[u^{e},\left\{t^{i e} \phi(i e)\right\}_{i \geq 1}\right]$. Then $\mathscr{S}$ is integral over $\mathscr{C}$, since $\left(t^{i} \phi(i)\right)^{e} \subseteq t^{i e} \phi(i e) \subseteq \mathscr{C}$ for all $i \geq 1$. Therefore $\mathscr{A}_{w}(\phi)=\mathscr{A}_{w}\left(\phi^{(e)}\right)$ and $\mathscr{E}(\phi)=\mathscr{E}\left(\phi^{(e)}\right)$ by (2.7.1). Also, $\phi^{(e)}=\{\phi(i e)\}_{i \geq 0}$ $=\left\{(\phi(e))^{i}\right\}_{i \geq 0}$ by $(2.2 .2)$, so (2.5.1) shows that $\mathscr{A}_{w}\left(\phi^{(e)}\right)=\hat{A}^{*}(\phi(e))$ and $\mathscr{E}\left(\phi^{(e)}\right)$ $=\mathscr{E}(\phi(e))$, so $\mathscr{A}_{w}(\phi)=\hat{A}^{*}(\phi(e))$ and $\mathscr{E}(\phi)=\mathscr{E}(\phi(e))$.

(2.8) is an interesting and useful application of (2.7.2).

(2.8) Corollary. Let $Q$ be a primary ideal in a Noetherian ring $R$ and assume that $\phi=\left\{Q^{(n)}\right\}_{n \geq 0}$ is a Noetherian on $R$. Then $\left(Q^{(n)}\right)_{w}$ is $\operatorname{Rad}(Q)$-primary for all $n \geq 1$.

Proof. (2.7.2) shows that $\mathscr{A}_{w}(\phi)=\hat{A}^{*}(\phi(e))$ for all $e \in \operatorname{Int}(\phi)$, and (2.5.3) shows that if $n$ is large, then $\hat{A}^{*}(\phi(e))=\operatorname{Ass}\left(R /\left((\phi(e))^{n}\right)_{a}\right)$. Therefore let $e \in$ Int $(\phi)$, let $n$ be large, and let $P \in \operatorname{Ass}\left(R /\left((\phi(e))^{n}\right)_{a}\right)$. Then $P \in \operatorname{Ass}\left(R /\left((\phi(e))^{m}\right)\right.$ for all large $m$, by [7, Proposition 3.4], and $(\phi(e))^{m}=\phi(m e)=Q^{(m e)}$, hence $P=\operatorname{Rad}(Q)$, q.e.d. 
Among other things, (2.9) extends (2.7.2) to $\Phi$ when each $\phi_{i}(1)$ has height at least one. (It should be noted that (2.9) (together with (2.3.2)) shows the somewhat surprising result that $\left\{p \cap R ; p \in \widehat{\mathscr{Q}}^{*}\left(\mathfrak{u}^{1} \mathscr{R}\right)\right\}=\{q \cap R ; q \in$ $\left.\hat{\mathscr{Q}}^{*}\left(u \mathscr{S}_{\mathrm{e}}\right)\right\}$ for all $e \in \operatorname{Int}(\Phi)$, where $\mathscr{R}=\mathscr{R}(R, \Phi)$ and $\mathscr{S}_{\mathrm{e}}=\mathscr{R}\left(R, \phi_{1}^{(e(1))} \cdots \phi_{g}^{(e(g))}\right)$. And a similar statement holds for (2.10).)

(2.9) Theorem. Let $R$ be a Noetherian ring, let $\Phi=\left(\phi_{1}, \cdots, \phi_{g}\right)$ be a collection of $g>1$ Noetherian filtrations on $R$ such that height $\left(\phi_{i}(1)\right) \geq 1$ for $i=1, \cdots, g$, and let $\mathrm{e}=\operatorname{Int}(\Phi)$ (see (2.1.8)). Then $\mathscr{A}_{w}(\Phi)=\hat{A}^{*}\left(\Phi^{(1)}(\mathrm{e})\right)$.

Proof. Let $\mathscr{R}=\mathscr{R}(R, \Phi)$ and let $\mathscr{B}=R\left[u_{1}^{\mathrm{e}(1)}, \cdots, u_{g}^{\mathrm{e}(g)},\left\{t_{1}^{i \mathrm{e}(1)} \phi_{1}(i \mathrm{e}(1))\right\}_{i \geq 1}\right.$, $\left.\cdots,\left\{t_{g}^{i e(g)} \phi_{g}(i e(g))\right\}_{i \geq 1}\right]$. Then $\mathscr{R}$ is integral over $\mathscr{R}$, since the definition of a filtration implies that $\left(t_{j}^{i} \phi_{j}(i)\right)^{\mathrm{e}(j)} \subseteq t_{j}^{i e(j)} \phi_{j}(i e(j)) \subseteq \mathscr{B}$ for all $i \geq 1$ and for $j=1, \cdots, g$, so it follows from $(2.7 .1)$ that $\mathscr{A}_{w}(\Phi)=\mathscr{A}_{w}\left(\Delta_{\mathrm{e}}\right)$, where $\Delta_{\mathrm{e}}=\left(\phi_{1}^{(\mathrm{e}(1))}, \cdots, \phi_{g}^{(\mathrm{e}(g))}\right)$.

For $j=1, \cdots, g$ let $I_{j}=\phi_{j}(e(j))\left(=\phi_{j}^{(\mathrm{e}(j))}(1)\right)$ and let $\mathscr{C}=R\left[u_{1}, \cdots, u_{g}\right.$, $\left.t_{1} I_{1}, \cdots, t_{g} I_{g}\right]$. Then (2.2.2) shows that $\phi_{j}(n e(j))=\left(\phi_{j}(e(j))^{n}=I_{j}^{n}\right.$ for $j=1$, $\cdots, g$ and for all $n \geq 1$, so $\mathscr{C}=\mathscr{R}\left(R, \Delta_{\mathrm{e}}\right)$, hence $\left\{p \cap R ; p \in \hat{\mathscr{Q}}^{*}\left(\mathfrak{u}^{1} \mathscr{R}\left(R, \Delta_{\mathrm{e}}\right)\right\}\right.$ $=\left\{p \cap R ; p \in \hat{A}^{*}\left(\mathfrak{u}^{1} \mathscr{C}\right)\right\}$ by (2.5.2). Now in [26, (2.1.4)], $\left\{p \cap R ; p \in \hat{A}^{*}\left(\mathfrak{u}^{1} \mathscr{C}\right)\right\}$ is defined to be $\hat{A}^{*}\left(I_{1}, \cdots, I_{g}\right)$ and it is shown in [26, (2.4)] that $\hat{A}^{*}\left(I_{1}, \cdots, I_{g}\right)$ $=\hat{A}^{*}\left(I_{1} \cdots I_{g}\right) \quad\left(\right.$ since height $\left(\phi_{i}(1)\right) \geq 1$ implies that height $\left(I_{i}\right) \geq 1$ for $i=1, \cdots, g)$. Therefore (2.3.2) implies that $\mathscr{A}_{w}\left(\Delta_{\mathrm{e}}\right)=\hat{A}^{*}\left(I_{1} \cdots I_{g}\right)$, hence $\mathscr{A}_{w}(\Phi)=\mathscr{A}_{w}\left(\mathcal{A}_{\mathrm{e}}\right)=\hat{A}^{*}\left(\phi_{1}(\mathrm{e}(1)) \cdots \phi_{g}(\mathrm{e}(\mathrm{g}))\right)=\hat{A}^{*}\left(\Phi^{(1)}(\mathrm{e})\right)$ by the preceding paragraph.

q.e.d.

Concerning (2.9) and its proof, it should be noted that the related conclusion $A_{w}(\Phi)=\hat{A}^{*}\left(\Phi^{(1)}(e)\right)$ for all $e \in \operatorname{Int}\left(\phi_{1} \cdots \phi_{g}\right)$ can be proved by using (2.6.2) and (2.7.2) (since these two results show that $\mathscr{A}_{w}(\Phi)=$ $\left.\mathscr{A}_{w}\left(\phi_{1} \cdots \phi_{g}\right)=\hat{A}^{*}\left(\left(\phi_{1} \cdots \phi_{g}\right)(e)\right)\right)$.

(2.10) is an essential prime divisor analog of (2.6) and (2.9). The part corresponding to (2.6) (that is, the subset part of (2.10)) is clearly weaker than (2.6), since we are not able to prove (even when $g=1$ ) an essential prime divisor analog of the equality $\mathscr{A}_{w}(\Phi)=\operatorname{Ass}\left(R /\left(\left(\Phi^{(1)}(\mathfrak{n})\right)_{w}\right)\right.$ for all large $\mathfrak{n} \in \mathscr{P}_{g}$. (One reason for this is that $\Phi^{(1)}(\mathfrak{n}) \rightarrow\left(\Phi^{(1)}(\mathfrak{n})\right)_{w}$ is a closure operation that satisfies a cancellation law, and we do not know a good "essential closure" analog of this.)

(2.10) Theorem. Let $R$ be a Noetherian ring, let $\Phi=\left(\phi_{1}, \cdots, \phi_{g}\right)$ be a collection of $g>1$ Noetherian filtrations on $R$ such that each $\phi_{i}(1)$ is 
regular, and let $e \in \operatorname{Int}(\Phi)$. Then $\mathscr{E}(\Phi)=\mathscr{E}\left(\Phi^{(1)}(e)\right) \subseteq\{P \in \operatorname{Spec}(R) ; P \in$ $\mathscr{E}\left(\Phi^{(1)}(\mathfrak{n})\right)$ for some $\left.\mathfrak{n} \in P_{g}\right\}$, and this containment may be proper.

Proof. Let $\mathscr{R}, \mathscr{B}, \Delta_{\mathrm{e}}, I_{j}=\phi_{j}(\mathrm{e}(j))=\phi_{j}^{(\mathrm{e}(j))}(1)$, and $\mathscr{C}$ be as in the proof of (2.9). Then $\mathscr{R}$ is integral over $\mathscr{B}$, so (2.7.1) shows that $\mathscr{E}(\Phi)=\mathscr{E}\left(\Delta_{\mathrm{e}}\right)$. Also, $\mathscr{C}=\mathscr{R}\left(R, \Delta_{\mathrm{e}}\right)$, hence $\left\{p \cap R ; p \in \mathscr{E}\left(\mathfrak{u}^{1} \mathscr{R}\left(R, \Delta_{\mathrm{e}}\right)\right\}=\left\{p \cap R ; p \in \mathscr{E}\left(\mathfrak{u}^{1} \mathscr{C}\right)\right\}\right.$. Now in [26, (2.1.4.)], $\left\{p \cap R ; p \in \mathscr{E}\left(\mathfrak{u}^{1} \mathscr{C}\right)\right\}$ is defined to be $\mathscr{E}\left(I_{1}, \cdots, I_{g}\right)$ and it is shown in [26, (2.5)] that $\mathscr{E}\left(I_{1}, \cdots, I_{g}\right)=\mathscr{E}\left(I_{1} \cdots I_{g}\right.$ ) (since each $I_{j}$ is regular if each $\left.\phi_{i}(1)\right)$ is). Therefore (2.3.4) implies that $\mathscr{E}\left(\Delta_{\mathrm{e}}\right)=\mathscr{E}\left(I_{1} \cdots I_{g}\right)$, hence $\mathscr{E}(\Phi)=\mathscr{E}\left(\phi_{1}(e(1)) \cdots \phi_{g}(e(g))\right)=\mathscr{E}\left(\Phi^{(1)}(e)\right)$.

To complete the proof of (2.10) note that it follows from what was just shown that $\mathscr{E}(\Phi) \subseteq\left\{P \in \operatorname{Spec}(R) ; P \in \mathscr{E}\left(\Phi^{(1)}(\mathfrak{n})\right)\right.$ for some $\left.\mathfrak{n} \in \mathscr{P}_{g}\right\}$, so it remains to show that this containment may be proper. For this, let $R=F[X, Y]$, where $F$ is a field and $X, Y$ are indeterminates, let $g=1$, and let $\phi$ be the filtration on $R$ such that $\phi(n)=(X Y)^{n} R$, if $n$ is even, and $\phi(n)=(X Y)^{n}(X, Y) R$, if $n$ is odd. Then (2.2.3) shows that $\phi$ is Noetherian, since it is readily checked that $\mathscr{R}(R, \phi)=R\left[u, t X Y(X, Y), t^{2}(X Y)^{2}\right]$, and it is clear that $2 \in \operatorname{Int}(\phi)$, so $\mathscr{E}(\phi)=\mathscr{E}\left(\phi^{(1)}(2)\right)$ by the preceding paragraph. Also, $R$ is locally unmixed and $\phi(2)$ is a regular principal ideal, so $[4,(2.4)]$ shows that $\hat{A}^{*}(\phi(2))=\mathscr{E}(\phi(2))=S$, where $S=\{P ; P$ is a minimal prime divisor of $\phi(2)\}$, and it is clear that $S=\{X R, Y R\}$ and that $\mathscr{E}(\phi(2))=\mathscr{E}\left(\phi^{(1)}(2)\right)$, hence it follows that $\mathscr{E}(\phi)=\mathscr{E}\left(\phi^{(1)}(2)\right)=\{X R, Y R\}$. Further, $\phi(1)$ is a normal ideal (that is, all its powers are integrally closed), so $\hat{A}^{*}(\phi(1))=\left\{P \in \operatorname{Spec}(R) ; P \in \operatorname{Ass}\left(R /\left((\phi(1))^{n}\right)_{a}\right)\right.$ for some $\left.n \geq 1\right\}$, by (2.5.3), $=\{X R, Y R,(X, Y) R\}$, and $\hat{A}^{*}(\phi(1))=\mathscr{E}(\phi(1))$ by [4, (2.4)] (since $R$ is locally unmixed). Therefore $\mathscr{E}(\phi)=\{X R, Y R\} \subset\{X R, Y R,(X, Y) R\}=\mathscr{E}(\phi(1))=$ $\mathscr{E}\left(\phi^{(1)}(1)\right) \subseteq\left\{P \in \operatorname{Spec}(R) ; P \in \mathscr{E}\left(\phi^{(1)}(n)\right)\right.$ for some $\left.n \geq 1\right\}$.

(2.11) is a corollary of (2.7.2), (2.9), and (2.10); it shows that $\mathscr{A}_{w}(\Phi)$ $=\mathscr{E}(\Phi)$ when $R$ is locally unmixed. (2.12) gives the quintasymptoticquintessential analog.

(2.11) Corollary. Assume that $R$ is a locally unmixed Noetherian ring. Then:

(2.11.1) If $\phi$ is a Noetherian filtration on $R$, then $\hat{A}^{*}(\phi(e))=\mathscr{A}_{w}(\phi)=$ $\mathscr{E}(\phi)=\mathscr{E}(\phi(e))$ for all $e \in \operatorname{Int}(\phi)$.

(2.11.2) If $\Phi=\left(\phi_{1}, \cdots, \phi_{g}\right)$ is a collection of $g>1$ Noetherian filtrations on $R$ such that $\phi_{i}(1)$ is regular, then $\hat{A}^{*}\left(\Phi^{(1)}(e)\right)=\mathscr{A}_{w}(\Phi)=\mathscr{E}(\Phi)=$ $\mathscr{E}\left(\Phi^{(1)}(e)\right)$ for all $e \in \operatorname{Int}(\Phi)$. 
Proof. For (2.11.2), if $R$ is locally unmixed, then it follows from [11, Corollary, p. 61] that $\mathscr{R}=\mathscr{R}(R, \Phi)$ is locally unmixed. Therefore $\hat{A}^{*}\left(\mathfrak{u}^{1} \mathscr{R}\right)=\mathscr{Q}\left(\mathfrak{u}^{1} \mathscr{R}\right)$, by $[4,(2.4)]$, and $\hat{A}^{*}\left(\mathfrak{u}^{1} \mathscr{R}\right)=\hat{\mathscr{Q}}^{*}\left(\mathfrak{u}^{1} \mathscr{R}\right)$, by $(2.5 .2)$, so $2\left(\mathfrak{u}^{1} \mathscr{R}\right)$ $=\widehat{\mathscr{Q}}^{*}\left(\mathfrak{u}^{1} \mathscr{R}\right)$. Thus it follows from (2.3.2) and (2.3.4) that $\mathscr{E}(\Phi)=\mathscr{A}_{w}(\Phi)$, and the other two equalities hold by (2.9), and (2.10).

The proof of (2.11.1) is similar, but use (2.7.2) in place of (2.9) and (2.10).

q.e.d.

(2.12) Remark. If $R$ is a locally unmixed Noetherian ring and if $\Phi=\left(\phi_{1}, \cdots, \phi_{g}\right)$ is a collection of $g \geq 1$ Noetherian filtrations on $R$, then $\widehat{\mathscr{Q}}^{*}\left(\Phi^{(1)}(\mathfrak{n})\right)=\widehat{\mathscr{Q}}^{*}(\Phi)=\mathscr{Q}(\Phi)=\mathscr{Q}\left(\Phi^{(1)}(\mathfrak{n})\right)$ for all $\mathfrak{n} \in \mathscr{P}_{g}$.

Proof. The hypothesis implies that $R_{P}$ is unmixed for all $P \in \operatorname{Spec}(R)$, so it follows from (2.3.1) and (2.3.3) that $\widehat{\mathscr{Q}}^{*}(I)=\mathscr{Q}(I)$ for all ideals $I$ in $R$. The conclusion follows from this and (2.4).

q.e.d.

(2.13) is another corollary of (2.9) and (2.10) (and its analogs for the case $g=f=1$ and for the quintasymptotic-quintessential case are given in (2.14)). For (2.13), it should be noted that the hypothesis $\Phi^{(\mathfrak{l})}(\mathfrak{l})=$ $\Gamma^{(\mathfrak{m})}(\mathfrak{n})$ together with (2.1.11) imply that $\operatorname{Rad}\left(\phi_{1} \cdots \phi_{g}\right)=\operatorname{Rad}\left(\gamma_{1} \cdots \gamma_{f}\right)$. Therefore the hypothesis that height $\left(\phi_{i}(1)\right) \geq 1$ in (2.13.1) (resp., $\phi_{i}(1)$ ) is regular in (2.13.2)) for $i=1, \cdots, g$ implies that each $\gamma_{i}(1)$ has height at least one in (2.13.1) (resp., that each $\gamma_{i}(1)$ is regular in (2.13.2)).

(2.13) Corollary. Let $\Phi=\left(\phi_{1}, \cdots, \phi_{g}\right)$ and $\Gamma=\left(\gamma_{1}, \cdots, \gamma_{f}\right)$ be finite collections of Noetherian filtrations on a Noetherian ring $R$ and assume that $\Phi^{(\mathfrak{h})}(\mathfrak{f})=\Gamma^{(\mathfrak{m})}(\mathfrak{n})$ for some $\mathfrak{h}, \mathfrak{l} \in \mathscr{P}_{g}$ such that $\mathfrak{h} \mathfrak{f}=\mathfrak{q e}$ for some $\mathfrak{q} \in \mathscr{P}_{g}$ and for some $\mathfrak{e} \in \operatorname{Int}(\Phi)$, and for some $\mathfrak{m}, \mathfrak{n} \in \mathscr{P}_{f}$ such that $\mathfrak{m n}=\mathfrak{q}^{\prime} \mathrm{e}^{\prime}$ for some $\mathfrak{q}^{\prime} \in \mathscr{P}_{f}$ and for some $\mathrm{e}^{\prime} \in \operatorname{Int}(\Gamma)$. Then:

(2.13.1) If height $\left(\phi_{i}(1)\right) \geq 1$ for $i=1, \cdots, g$, then $\mathscr{A}_{w}(\Phi)=\hat{A}^{*}\left(\Phi^{(1)}(e)\right)$ $=\hat{A}^{*}\left(\Gamma^{(1)}\left(e^{\prime}\right)\right)=\mathscr{A}_{w}(\Gamma)$ for all $\mathrm{e} \in \operatorname{Int}(\Phi)$ and for all $\mathrm{e}^{\prime} \in \operatorname{Int}(\Gamma)$.

(2.13.2) If $\phi_{i}(1)$ is a regular ideal for $i=1, \cdots, g$, then $\mathscr{E}(\Phi)=\mathscr{E}\left(\Phi^{(1)}(\mathfrak{e})\right)$ $=\mathscr{E}\left(\Gamma^{(1)}\left(\mathfrak{e}^{\prime}\right)\right)=\mathscr{E}(\Gamma)$ for all $e \in \operatorname{Int}(\Phi)$ and for all $e^{\prime} \in \operatorname{Int}(\Gamma)$.

Proof. For (2.13.1), (2.1.11) (a)-(b) show that $\Phi^{(\mathfrak{b})}(\mathfrak{l})=\Phi^{(1)}(\mathfrak{h} \mathfrak{f})$. Also, it is readily checked that if $e \in \operatorname{Int}(\Phi)$, then so is ne for all $\mathfrak{n} \in \mathscr{P}_{g}$. Therefore $\mathfrak{h} \mathfrak{f}=\mathfrak{q} e \in \operatorname{Int}(\Phi)$, by hypothesis, and $\mathscr{A}_{w}(\Phi)=\hat{A}^{*}\left(\Phi^{(1)}(e)\right)$ for all $e \in \operatorname{Int}(\Phi)$ by (2.9), so $\hat{A}^{*}\left(\Phi^{(1)}\left(\mathfrak{h}_{\mathfrak{l}}\right)\right)=\mathscr{A}_{w}(\Phi)$. And it follows similarly that $\hat{A}^{*}\left(\Gamma^{(1)}(\mathfrak{m n})\right)$ $=\mathscr{A}_{w}(\Gamma)=\hat{A}^{*}\left(\Gamma^{(\mathfrak{1})}\left(\mathfrak{e}^{\prime}\right)\right)$. (2.13.1) follows from this since $\Phi^{(1)}(\mathfrak{h} \mathfrak{l})=\Phi^{(\mathfrak{h})}(\mathfrak{f})=$ $\Gamma^{(\mathfrak{m})}(\mathfrak{n})=\Gamma^{(1)}(\mathfrak{m} \mathfrak{n})$. 
The proof of (2.13.2) is similar, but use (2.10) in place of (2.9). q.e.d.

(2.14) gives the analogs of (2.13) for the case $g=f=1$ and for the quintasymptotic-quintessential case.

(2.14) Corollary. Let $\Phi=\left(\phi_{1}, \cdots, \phi_{g}\right)$ and $\Gamma=\left(\gamma_{1}, \cdots, \gamma_{f}\right)$ be finite collections of Noetherian filtrations on a Noetherian ring $R$ and assume that $\Phi^{(\mathfrak{l})}(\mathfrak{f})=\Gamma^{(\mathfrak{m})}(\mathfrak{n})$ for some $\mathfrak{h}, \mathfrak{f} \in \mathscr{P}_{g}$ and for some $\mathfrak{m}, \mathfrak{n} \in \mathscr{P}_{f}$. Then:

(2.14.1) $\hat{\mathscr{Q}}^{*}(\Phi)=\widehat{\mathscr{Q}}^{*}\left(\Phi^{(1)}(\mathfrak{n})\right)=\hat{\mathscr{Q}}^{*}\left(\Gamma^{(1)}(\mathfrak{n})\right)=\hat{\mathscr{Q}}^{*}(\Gamma)$ for all $\mathfrak{n} \in \mathscr{P}_{g}$.

(2.14.2) $\mathscr{2}(\Phi)=\mathscr{2}\left(\Phi^{(1)}(\mathfrak{n})\right)=\mathscr{2}\left(\Gamma^{(1)}(\mathfrak{n})\right)=\mathscr{2}(\Gamma)$ for all $\mathfrak{n} \in \mathscr{P}_{g}$.

(2.14.3) If $\Phi=(\phi)$ and $\Gamma=(\gamma)$, if $\mathfrak{h}=\mathfrak{q}=$ for some $\mathfrak{q} \geq 1$ and for some $e \in \operatorname{Int}(\phi)$, and if $\mathfrak{m n}=\mathfrak{q}^{\prime} \mathrm{e}^{\prime}$ for some $\mathfrak{q}^{\prime} \geq 1$ and for some $\mathrm{e}^{\prime} \in \operatorname{Int}(\gamma)$, then $\left.\mathscr{A}_{w}(\phi)=\hat{A}^{*}(\phi(e))=\hat{A}^{*}\left(\gamma\left(e^{\prime}\right)\right)=\mathscr{A}_{w}(\gamma)\right)$ and $\mathscr{E}(\phi)=\mathscr{E}(\phi(e))=\mathscr{E}\left(\gamma\left(e^{\prime}\right)\right)=\mathscr{E}(\gamma)$ for all $e \in \operatorname{Int}(\phi)$ and for all $e^{\prime} \in \operatorname{Int}(\gamma)$.

Proof. (2.1.11) (a)-(b) show that $\Phi^{(\mathfrak{b})}(\mathfrak{l})=\Phi^{(1)}(\mathfrak{h} \mathfrak{f})$ and that $\Gamma^{(\mathrm{m})}(\mathfrak{n})=$ $\Gamma^{(1)}(\mathfrak{m})$. (2.14.1) and (2.14.2) follow immediately from this, the hypothesis that $\Phi^{(\mathfrak{k})}(\mathfrak{l})=\Gamma^{(\mathrm{m})}(\mathfrak{n})$, and $(2.4)$.

The proof of (2.14.3) is similar to the proof of (2.13.1), but use (2.7.2) in place of (2.9).

q.e.d.

It turns out in the ideal case that the asymptotic and essential prime divisors are quite a bit more useful than the quintasymptotic and quintessential prime divisors. We think this will also be true for Noetherian filtrations, so since the essential prime divisors of $\Phi$ are new to this paper we will prove one more result concerning them.

(2.15) Theorem. Let $\Phi=\left(\phi_{1}, \cdots, \phi_{g}\right)$ be a finite collection of Noetherian filtrations on a Noetherian ring $R$ such that each $\phi_{i}(1)$ is regular and let $\mathrm{e} \in \operatorname{Int}(\Phi)$. Then:

(2.15.1) If $P \in \mathscr{E}\left(\Phi^{(1)}(\mathfrak{q e})\right)$ for some $\mathfrak{q} \in \mathscr{P}_{g}$, then $P \in \mathscr{E}\left(\Phi^{(1)}(\mathfrak{q} e+\mathfrak{n})\right)$ for all $\mathfrak{n} \geq e$.

(2.15.2) If $P \in \mathscr{E}\left(\Phi^{(1)}(\mathfrak{n})\right)$ for some $\mathfrak{n} \geq e$, then $P \in \mathscr{E}\left(\Phi^{(1)}(\mathfrak{q} e+\mathfrak{n})\right)$ for all $q \in \mathscr{P}_{g}$.

Proof. It is shown in [21, (4.3)] that if $I$ and $J$ are regular ideals in a Noetherian ring $R$ and if $P \in \mathscr{E}(I)$, then $P \in \mathscr{E}(I J)$, so both parts follow readily from this and the definition of $\operatorname{Int}(\Phi)$ (see (2.1.8)). q.e.d.

Let \# denote any one of: quintasymptotic; quintessential; asymptotic; or, essential. Then because of (2.7.2), (2.9), and (2.10), a number of theo- 
rems for the \#-prime divisors of ideals could now be extended to finite collections of Noetherian filtrations (and, as a special case, to finite collections of ideals). For example, the \#-prime divisors of $\Phi$ behave nicely when passing to localizations, factor rings modulo prime divisors of zero, finite integral extension rings, and faithfully flat Noetherian extension rings. However, we will not list these results here but will, instead, now consider \#-sequences over $\Phi$.

\section{§3. Asymptotic sequenes, grade, and cograde for a Noetherian filtration}

As an application of the results of Section 2 (especially (2.7.2)), in this section we introduce the four types of sequences (corresponding to the four types of prime divisors considered in Section 2) in (and, over) a collection $\Phi=\left(\phi_{1}, \cdots, \phi_{g}\right)$ of $g \geq 1$ Noetherian filtrations and prove a few of their basic properties. Among these is that they are the same type of sequence in (and, over) $\Phi^{(1)}(e)$ for each $e \in \cap\left\{\operatorname{Int}\left(\phi_{i}\right) ; i=1, \cdots, g\right\}$ and, as in the ideal case, that they lead naturally to the four corresponding grades and cogrades of $\Phi$.

Notational Convention. Throughout this section we let \# denote any one of: quintasymptotic; asymptotic; quintessential; or, essential. (Here, the same word is to be substituted throughout each result.)

(3.1) Definition. Let $I$ be an ideal in a Noetherian ring $R$, let $\Phi=$ $\left(\phi_{1}, \cdots, \phi_{g}\right)$ be a collection of $g \geq 1$ Noetherian filtrations on $R$, let $x_{1}$, $\cdots, x_{h}$ be elements in $R$, let $\chi_{0}$ be the zero filtration (each ideal in the sequence is the zero ideal of $R$ ), and for $j=1, \cdots, h$ let $\chi_{j}=\left\{\left(x_{1}, \cdots, x_{j}\right)^{n} R\right\}_{n \geq 0}$. Also, let $\mathscr{P}_{\#}(I)$ (resp., $\mathscr{P}_{\sharp}(\Phi)$ ) denote the set of $\#$-prime divisors of $I$ (resp., $\Phi)$. Then:

(3.1.1.) $x_{1}, \cdots, x_{h}$ are a $\sharp$-sequence over $I$ (resp., $\left.\Phi\right)$ in case $\left(I, x_{1}, \cdots\right.$, $\left.x_{h}\right) R \neq R$ (resp., $\left(\Phi^{(1)}(1), x_{1}, \cdots, x_{h}\right) R \neq R$ ) and $x_{j+1} \notin \cup\left\{P ; P \in \mathscr{P}_{\sharp}\left(I, x_{1}, \cdots\right.\right.$, $\left.\left.x_{j}\right)\right\} \quad$ (resp., $\quad x_{j+1} \notin \cup\left\{P ; P \in \mathscr{P}_{\#}\left(\Phi^{(1)}+\chi_{j}\right)\right\}$ ) for $j=0,1, \cdots, h-1$. A \#sequence over the zero ideal (resp., filtration) is called a \#-sequence in $R$.

(3.1.2) The \#-grade of $I$ (resp., $\Phi$ ) is the length of a \#-sequence in $R$ that is maximal with respect to being contained in $I$ (resp., $\Phi^{(1)}(1)$ ).

(3.1.3) If $R$ is loacl, then the $\#$-cograde of $I$ (resp., $\Phi)$ is the length of a maximal \#-sequence over $I$ (resp., $\Phi)$. 
Concerning (3.1.2) and (3.1.3), it is known that the \#-grade and \#cograde of an ideal $I$ are well defined. Specifically: for $\#=$ 'asymptotic', these are shown in [17, (3.1)] and [3], respectively; for \#= 'essential', see [4, (3.10) and (4.1)]; for \#= 'quintessential', see [9, (5.3)] and [23, (3.2)]; and for \#= 'quintasymptotic', the results in [10] can be used (however, quintasymptotic grade and cograde are not specifically mentioned in [10]). This will be used in this section to show that the \#-grade and \#-cograde of $\Phi$ are also well defined.

And it should be noted that, among other things, the results in this section show that if $g=1$, if $\Phi=(\phi)$, and if $\phi=\left\{I^{n}\right\}_{n \geq 0}$ is the sequence of powers of an ideal $I$, then the three definitions in (3.1) agree for $I$ and for $\Phi$. In fact, this follows from (3.2) for (3.1.2), and (3.3) (resp., (3.5)) show that this holds for (3.1.1) (resp., (3.1.3)) since $1 \in \operatorname{Int}(\phi)$.

Our first result concerning these definitions is a special case of a more general result, but it is still of sufficient interest and use to warrant being specifically stated; it shows that each of the various grades of $\Phi$ defined in (3.1.2) is the corresponding grade of each of the ideals $\Phi^{(1)}(\mathfrak{n})$ for all $\mathfrak{n} \in \mathscr{P}_{g}$.

(3.2) Theorem. With the notation of (3.1) let $\mathfrak{n} \in \mathscr{P}_{g} . \quad$ Then $\sharp$-grade $(\Phi)$ $=\#-\operatorname{grade}\left(\Phi^{(1)}(\mathfrak{n})\right)$.

Proof. Let $I$ be an ideal in $R$ and let $b_{1}, \cdots, b_{h}$ be a \#-sequence in $R$ that is maximal with respect to being contained in $I$ (so \#-grade $(I)=h$ by the paragraph following (3.1)). Then necessarily $I \subseteq Q$ for some $Q \in$ $\mathscr{P}_{\#}\left(\left(b_{1}, \cdots, b_{h}\right) R\right)$, so it follows that $\#$-grade $(Q)=h$. Therefore $h=\#$-grade $(I)$ $\leq \#$-grade $(\operatorname{Rad}(I)) \leq \#$-grade $(Q)=h$, so \#-grade $(I)=\#$-grade $(\operatorname{Rad}(I))$. The conclusion follows from this and (3.1.2), since $\operatorname{Rad}\left(\Phi^{(1)}(1)\right)=\operatorname{Rad}\left(\Phi^{(1)}(\mathfrak{n})\right)$.

Before proving the \#-cograde analog of (3.2) we first prove a stronger result concerning \#-sequences over $\Phi$.

(3.3) THeOREM. With the notation of (3.1), $x_{1}, \cdots, x_{h}$ are a \#-sequence over $\Phi$ if and only if they are $a \#$-seqence over $\Phi^{(1)}(e)$ for each $e \in \cap\left\{\operatorname{Int}\left(\phi_{i}\right)\right.$; $i=1, \cdots, g\}$.

Proof. (Note first that there exists a positive integer $e$ in $\cap\left\{\operatorname{Int}\left(\phi_{i}\right)\right.$; $i=1, \cdots, g\}$, since (2.2.3) shows that there exists a positive integer $e_{i}$ in $\operatorname{Int}\left(\phi_{i}\right)$ for $i=1, \cdots, g$, and then a brief computation shows that $e_{1} \cdots e_{g}$ 
$\epsilon \operatorname{Int}\left(\phi_{i}\right)$ for $i=1, \cdots, g$.)

Assume first that \# denotes 'quintasymptotic'. Then by (3.3.1) it suffices to show that $\widehat{\mathscr{Q}}^{*}\left(\Phi^{(1)}+\chi_{j}\right)=\widehat{\mathscr{Q}}^{*}\left(\left(\Phi^{(1)}(e), x_{1}, \cdots, x_{j}\right) R\right)$ for $j=0,1$, $\cdots, h-1$. For this, as noted in the proof of (2.4), if $I$ and $J$ are ideals such that $\operatorname{Rad}(I)=\operatorname{Rad}(J)$, then $\hat{\mathscr{Q}}^{*}(I)=\hat{\mathscr{Q}}^{*}(J)$. Therefore for $j=0$, $1, \cdots, h-1$ it follows that $\hat{\mathscr{Q}}^{*}\left(\Phi^{(1)}+\chi_{j}\right)=\widehat{\mathscr{Q}}^{*}\left(\left(\Phi^{(1)}+\chi_{j}\right)(1)\right)$, by $(2.3 .1),=$ $\hat{\mathscr{Q}}^{*}\left(\Phi^{(1)}(1)+\chi_{j}(1)\right)$, by $\left.(2.1 .4),=\hat{\mathscr{Q}}^{*}\left(\Phi^{(1)}(1), x_{1}, \cdots, x_{j}\right) R\right)=\hat{\mathscr{Q}}^{*}\left(\Phi^{(1)}(e), x_{1}, \cdots\right.$, $\left.\left.x_{j}\right) R\right)$, since $\operatorname{Rad}\left(\Phi^{(1)}(1)\right)=\operatorname{Rad}\left(\Phi^{(1)}(e)\right)$. Therefore $\widehat{\mathscr{Q}}^{*}\left(\Phi^{(1)}+\chi_{j}\right)=\widehat{\mathscr{Q}}^{*}\left(\Phi^{(1)}(e)\right.$, $\left.x_{1}, \cdots, x_{j}\right) R$ ), so the conclusion holds in this case, and a similar computation (using (2.3.3) in place of (2.3.1)) shows that it also holds when \# denotes 'quintessential'.

If $\#$ denotes 'essential', then it is shown in $[4,(3.11 .1)]$ that $x_{1}, \cdots, x_{h}$ are a \#-sequence over an ideal $I$ if and only if $x_{1}^{n_{1}}, \cdots, x_{h}^{n_{h}}$ are for some positive integers $n_{i}$ if and only if $x_{1}^{n_{1}}, \cdots, x_{h}^{n_{h}}$ are for all positive integers $n_{i}$, and if \# denotes 'asymptotic', then the analogous result also holds. (We do not know a reference for this, but its proof is similar to the proof of (3.11.1) in [4] (but use [20, (5.6.1)] and [17, (3.16)] in place of [4, (3.9)] and $([23,(2.11 .1)]$, respectively). $)$ Therefore when \# denotes either 'asymptotic' or 'essential' it suffices to show that $x_{1}, \cdots, x_{h}$ are a \#-sequence over $\Phi$ if and only if $x_{1}^{e}, \cdots, x_{h}^{e}$ are a $\#$-sequence over $\Phi^{(1)}(e)$.

For this, for $j=0,1, \cdots, h-1$ let $\mathscr{R}_{j}=\mathscr{R}\left(R, \Phi^{(1)}+\chi_{j}\right)$ and $\mathscr{B}_{j}=$ $R\left[u^{e}, t^{e}\left(\Phi^{(1)}(e), x_{1}^{e}, \cdots, x_{j}^{e}\right) R\right]$. Then since $\left(t^{m} \Phi^{(1)}(m)\right)^{e} \subseteq t^{m e} \Phi^{(1)}(m e)=\left(t^{e} \Phi^{(1)}(e)\right)^{m}$ $\subseteq \mathscr{B}_{j}$ for all $m \geq 1$ (since $e \in \cap\left\{\operatorname{Int}\left(\phi_{i}\right) ; i=1, \cdots, g\right\}$ ), it follows that $\mathscr{R}_{j}$ is integral over $\mathscr{B}_{j}$, so if \# denotes either 'asymptotic' or 'essential', then it follows from (2.7.1) (with $g=1$ and $\gamma_{1}$ the filtration such that $\gamma_{1}(q e+i)$ $=\left(\Phi^{(1)}(e), x_{1}^{e}, \cdots, x_{j}^{e}\right)^{q+1} R$ for $q \geq 0$ and for $\left.i=1, \cdots, e\right)$ that $\mathscr{A}_{w}\left(\Phi^{(1)}+\chi_{j}\right)$ $=\mathscr{A}_{w}\left(\gamma_{1}^{(e)}\right)$ and $\mathscr{E}\left(\Phi^{(1)}+\chi_{j}\right)=\mathscr{E}\left(\gamma_{1}^{(e)}\right)$, and (2.5.1) shows that $\mathscr{A}_{w}\left(\gamma_{1}^{(e)}\right)=$ $\hat{A}^{*}\left(\left(\Phi^{(1)}(e), x_{1}^{e}, \cdots, x_{j}^{e}\right) R\right)$ and $\mathscr{E}\left(\gamma_{1}^{(e)}\right)=\mathscr{E}\left(\left(\Phi^{(1)}(e), x_{1}^{e}, \cdots, x_{j}^{e}\right) R\right)\left(\right.$ since $\gamma_{1}^{(e)}(n)=$ $\left(\Phi^{(1)}(e), x_{1}^{e}, \cdots, x_{j}^{e}\right)^{n} R$ for all $\left.n \geq 1\right)$. Therefore the conclusion holds in these two cases also.

q.e.d.

(3.4) Remark. (3.4.1) It follows as in the second paragraph of the proof of (3.3) that if \# denotes either 'quintasymptotic' or 'quintessential', then $x_{1}, \cdots, x_{h}$ are a $\#$-sequence over $\Phi$ if and only if they are a $\#$-sequence over $\Phi^{(1)}(\mathfrak{n})$ for all (equivalently, for some) $\mathfrak{n} \in \mathscr{P}_{g}$.

(3.4.2) If $\#$ is either 'asymptotic' or 'essential', and if $x_{1}, \cdots, x_{h}$ are a \#-sequence over $\Phi$, then $x_{1} \notin \cup\left\{P \in \mathscr{P}_{\#}\left(\phi_{i}\right) ; i=1, \cdots, g\right\}$, so if each permutation of $x_{1}, \cdots, x_{h}$ is a \#-sequence over $\Phi$ (this holds when $R$ is local, 
by $[20,(5.8)$ and (5.6.1)] (for asymptotic) and [23, (6.2)] (for essential)), then $x_{j} \notin \cup\left\{P \in \mathscr{P}_{\#}\left(\phi_{i}\right) ; i=1, \cdots, g\right\}$ for $j=1, \cdots, h$.

Proof of (3.4.2). Let $\mathscr{R}_{i}=R\left[u_{i},\left\{t_{i}^{n} \phi_{i}(n)\right\}_{n=1}^{\infty}\right], \mathscr{R}=\mathscr{R}(R, \Phi)$, and $\mathscr{T}=$ $\mathscr{R}_{i}\left[u_{1}, \cdots, u_{i-1}, u_{i+1}, \cdots, u_{g}, t_{1}, \cdots, t_{g}\right]$. Then $\mathscr{R}_{i} \subseteq \mathscr{R} \subseteq \mathscr{T}, \mathscr{T}=\mathscr{R}\left[1 / u_{1} \cdots u_{g}\right]$ is a localization of $\mathscr{R}$, and $\mathscr{T}$ is a localization of a pure transcendental extension ring of $\mathscr{R}_{i}$. Therefore assume that \# denotes 'asymptotic' and let $P \in \mathscr{A}_{w}\left(\phi_{i}\right)$. Then there exists $p \in \widehat{\mathscr{Q}}^{*}\left(u_{i} \mathscr{R}_{i}\right)$ such that $p \cap R=P$, and then $q=p \mathscr{T} \cap \mathscr{R} \in \widehat{\mathscr{Q}}^{*}\left(u_{i} \mathscr{R}\right)$, by $\left[10,(3.4)\right.$ and (3.6)], so since each $u_{j}$ is regular it follows that $q \in \widehat{\mathscr{Q}}^{*}\left(u_{1} \cdots u_{g} \mathscr{R}\right)$. Therefore $P=q \cap R \in \mathscr{A}_{w}(\Phi)$ by (2.3.2), so $\mathscr{A}_{w}\left(\phi_{i}\right) \subseteq \mathscr{A}_{w}(\Phi)$. The case when \# denotes 'essential' is proved similarly, but use [4, (2.5.1) and (2.5.3)] in place of $[10,(3.4)$ and (3.6)].

q.e.d.

(3.5) CoROLlaRY. With the notation of (3.3) assume that $R$ is a local ring. Then $\#$-cograde $(\Phi)=\#$-cograde $\left(\Phi^{(1)}(e)\right)$ for all $e \in \cap\left\{\operatorname{Int}\left(\phi_{i}\right) ; i=1\right.$, $\cdots, g\}$.

Proof. This follows immediately from (3.3) and (3.1.3) (together with the fact, noted in the paragraph following (3.1), that the $\#$-cograde of ideals in $R$ is well defined).

q.e.d.

Because of (3.2), (3.3), and (3.5), a number of theorems concerning $\#$-sequences in (and, over) an ideal, as well as the \#-grade and \#-cograde of an ideal, could now be extended to finite collections of Noetherian filtrations (and to finite collections of ideals). For example, they behave nicely when passing to the same types of rings as mentioned at the end of Section 2. However, we will not list these results here but will, instead, now consider the application of the results in this section to the analytic spread of $\Phi$.

\section{§4. On the analytic spread of a Noetherian filtration}

In this section, by using the results in Section 3, we extend a couple of important theorems concerning the analytic spread of an ideal in a local ring $R$ to the analytic spread of a finite collection of Noetherian filtrations on $R$. We begin with the definition of analytic spread.

(4.1) Definition. Let $(R, M)$ be a local ring, let $I$ be an ideal in $R$, and let $\Phi=\left(\phi_{1}, \cdots, \phi\right)$ be a collection of $g \geq 1$ Noetherian filtrations on $R$. Then: 
(4.1.1) The analytic spread a $(I)$ of $I$ is the integer $\mathrm{a}(I)=\operatorname{altitude}(\mathscr{R} \mid$ $(u, M) \mathscr{R})$, where $\mathscr{R}=\mathscr{R}(R, I)$.

(4.1.2) The analytic spead a $(\Phi)$ of $\Phi$ is the integer a $(\Phi)=$ altitude $(\mathscr{R} /$ $(u, M) \mathscr{R})$, where $\mathscr{R}=\mathscr{R}\left(R, \Phi^{(1)}\right)$.

It should be noted that if $\Phi=(\phi)$ and $\phi=\left\{I^{n}\right\}_{n \geq 0}$ is the sequence of powers of an ideal $I$, then (4.1.1) and (4.1.2) agree; this follows from (4.2.2) (Since $1 \in \operatorname{Int}(\phi)$ ). In fact, (4.2.2) shows the stronger result that if $e \in \cap\left\{\operatorname{Int}\left(\phi_{i}\right) ; i=1, \cdots, g\right\}$, then the analytic spread of $\Phi$ is the analytic spread of the ideal $\Phi^{(1)}(e)$.

(4.2) Theorem. Let $\Phi=\left(\phi_{1}, \cdots, \phi_{g}\right)$ be a finite collection of Noetherian fitrations on a local ring $R$. Then:

(4.2.1) If $\Gamma=\left(\gamma_{1}, \cdots, \gamma_{f}\right)$ is another finite collection of Noetherian filtrations on $R$ such that $\mathscr{R}=\mathscr{R}\left(R, \Phi^{(1)}\right)$ is an integral extension ring of $\mathscr{B}=R\left[u^{n}, t^{n} \Gamma^{(1)}\right]$ for some $n \geq 1$, then $a(\Phi)=a(\Gamma)$.

(4.2.2) $a(\Phi)=a\left(\Phi^{(1)}(e)\right)$ for all $e \in \cap\left\{\operatorname{Int}\left(\phi_{i}\right) ; i=1, \cdots, g\right\}$.

Proof. For (4.2.1), if $\mathscr{R}$ is an integral extension ring of $\mathscr{B}$, then $\left(u^{n}, M\right) \mathscr{B} \subseteq\left(u^{n}, M\right) \mathscr{R} \cap \mathscr{B} \subseteq\left(\left(u^{n}, M\right) \mathscr{B}\right)_{a}$, the integral closure of $\left(u^{n}, M\right) \mathscr{B}$, so it follows that altitude $\left(\mathscr{B} /\left(u^{n}, M\right) \mathscr{B}\right)=$ altitude $\left(\mathscr{B} /\left(\left(u^{n}, M\right) \mathscr{R} \cap \mathscr{B}\right)\right)$. Also, altitude $\left(\mathscr{B} /\left(\left(u^{n}, M\right) \mathscr{R} \cap \mathscr{R}\right)\right)=$ altitude $\left(\mathscr{R} /\left(\mathfrak{l t}^{n}, M\right) \mathscr{R}\right)$, by integral dependence, and altitude $\left(\mathscr{R} /\left(\mathfrak{u}^{n}, M\right) \mathscr{R}\right)=$ altitude $(\mathscr{R} /(u, M) \mathscr{R})=a(\Phi)$ by (4.1.2). Further, $\mathscr{B} \cong \mathscr{R}\left(R, \Gamma^{(1)}\right)$, so altitude $\left(\mathscr{B} /\left(u^{n}, M\right) \mathscr{B}\right)=a(\Gamma)$ by (4.1.2) and the isomorphism, so it follows that $a(\Phi)=a(\Gamma)$.

For (4.2.2) let $e \in \cap\left\{\operatorname{Int}\left(\phi_{i}\right) ; i=1, \cdots, g\right\}$ (see the start of the proof of (3.3)) and let $\left.\mathscr{C}=R\left[u^{e}, t^{e} \Phi^{(1)}(e)\right)\right]$. Then it follows as in the last paragraph of the proof of (3.3) that $\mathscr{R}$ is integral over $\mathscr{C}$. Also, $\mathscr{C} \cong$ $\mathscr{R}\left(R, \Phi^{(1)}(e)\right)=\mathscr{R}(R, \gamma)=\mathscr{R}\left(R, \gamma^{(1)}\right)$, where $\gamma=\left\{\left(\Phi^{(1)}(e)\right)^{n}\right\}_{n \geq 0}$. Therefore $a(\Phi)$ $=a(\gamma)$, by (4.2.1), and $a(\gamma)=a\left(\Phi^{(1)}(e)\right)$ by (4.1.1). q.e.d.

It is a fairly important result that adding an asymptotic sequence over an ideal $I$ to $I$ increases the analytic spread by the length of the sequence. (4.3) shows that this continues to hold for $\Phi$.

(4.3) Corollary. With the notation of (4.2) assume that $x_{1}, \cdots, x_{h}$ are an asymptotic sequence over $\Phi$ and let $\chi=\left\{\left(x_{1}, \cdots, x_{h}\right) R\right\}_{n \geq 0}$. Then $a(\Phi+\chi)=a(\Phi)+h$.

Proof. Let $\mathscr{R}=\mathscr{R}\left(R,(\Phi+\chi)^{(1)}\right)$, let $e \in \cap\left\{\operatorname{Int}\left(\phi_{i}\right) ; i=1, \cdots, g\right\}$, and let $\mathscr{B}=R\left[u^{e}, t^{e}\left(\Phi(e), x_{1}^{e}, \cdots, x_{h}^{e}\right) R\right]$. Then $\mathscr{B} \cong \mathscr{R}\left(R,\left(\Phi^{(1)}(e), x_{1}^{e}, \cdots, x_{h}^{e}\right) R\right)=$ 
$\mathscr{R}(R, \gamma)=\mathscr{R}\left(R, \gamma^{(1)}\right)$, where $\gamma=\left\{\left(\Phi^{(1)}(e), x_{1}^{e}, \cdots, x_{h}^{e}\right)^{n} R\right\}_{n \geq 0}$. Also, it follows as in the last paragraph of the proof of (3.3) that $\mathscr{R}$ is integral over $\mathscr{B}$, so (4.2.1) shows that $a(\Phi+\chi)=a(\gamma)$, and $a(\gamma)=a\left(\Phi^{(1)}(e), x_{1}^{e}, \cdots, x_{h}^{e}\right) R$ ) by (4.1.1) and (4.1.2) (since $\mathscr{R}\left(R, \gamma^{(1)}\right)=\mathscr{R}(R, \gamma)=\mathscr{R}\left(R,\left(\Phi^{(1)}(e), x_{1}^{e}, \cdots, x_{h}^{e}\right) R\right)$ ). Further, $x_{1}, \cdots, x_{h}$ are an asymptotic sequence over $\Phi^{(1)}(e)$, by hypothesis and (3.3), so $x_{1}^{e}, \cdots, x_{h}^{e}$ are (as noted in the proof of (3.3)). Therefore $\left.a\left(\Phi^{(1)}(e), x_{1}^{e}, \cdots, x_{h}^{e}\right) R\right)=a\left(\Phi^{(1)}(e)\right)+h$, by $[8,(3.1)],=a(\Phi)+h$ by (4.2.2), hence $a(\Phi+\chi)=a(\Phi)+h$.

q.e.d.

(4.4) extends to $\Phi$ the following theorem of Rees [27, (3.3)]: a $(I)+$ $\operatorname{acogd}(I)=\operatorname{altitude}(R)$ for all ideal $I$ in a quasi-unmixed local ring $R$. (Here, acogd denoted asymptotic cograde.)

(4.4) Corollary. With the notation of (4.2) assume that $R$ is quasi. unmixed. Then $\mathrm{a}(\Phi)+\operatorname{acogd}(\Phi)=$ altitude $(R)$.

Proof. Let $e \in \cap\left\{\operatorname{Int}\left(\phi_{i}\right) ; i=1, \cdots, g\right\}$. Then $\mathrm{a}(\Phi)=\mathrm{a}\left(\Phi^{(1)}(e)\right)$, by (4.2.2), and $\operatorname{acogd}(\Phi)=\operatorname{acogd}\left(\Phi^{(1)}(e)\right)$, by (3.5), so a $(\Phi)+\operatorname{acogd}(\Phi)=\mathrm{a}\left(\Phi^{(1)}(e)\right)$ $+\operatorname{acogd}\left(\Phi^{(1)}(e)\right)=\operatorname{altitude}(R)$ by [27, (3.3)].

q.e.d.

Our final result shows that the formula in (4.4) characterizes when $R$ is quasiunmixed.

(4.5) Corollary. If $R$ is a local ring such that $\mathrm{a}(\Phi)+\operatorname{acogd}(\Phi)=$ altitude $(R)$ for all collections of $g \geq 1$ Noetherian filtrations on $R$, then $R$ is quasi-unmixed.

Proof. Let $I$ be an ideal in $R$ and let $\gamma=\left\{I^{n}\right\}_{n \geq 0}$. Then $1 \in \operatorname{Int}(\gamma)$ and $\gamma^{(1)}(1)=I$, so (4.2.2) shows that $\mathrm{a}(\gamma)=\mathrm{a}\left(\gamma^{(1)}(1)\right)=\mathrm{a}(I)$, and (3.5) shows that $\operatorname{acogd}(\gamma)=\operatorname{acogd}\left(\gamma^{(1)}(1)\right)=\operatorname{acogd}(I)$. Therefore the conclusion follows from [18, (4.4)] which shows that $R$ is quasi-unmixed if a $(I)+\operatorname{acogd}(I)$ $=$ altitude $(R)$ for all ideals $I$ in $R$.

q.e.d.

\section{REFERENCES}

[1] W. Bishop, J. W. Petro, L. J. Ratliff, Jr. and D. E. Rush, Note on Noetherian filtrations, Comm. Algebra, 17 (1989), 471-485.

[2] N. Bourbaki, Elements of Mathematics; Commutative Algebra, Addison-Wesley, Reading, Mass., 1972.

[ 3 ] D. Katz, A note on asymptotic prime sequences, Proc. Amer. Math. Soc., 87 (1983), 415-418.

[ 4 ] — and L. J. Ratliff, Jr., $U$-essential prime divisions and sequences, Nagoya Math. J., 103 (1986), 39-66.

[ 5 ] — S. McAdam, J. S. Okon and L. J. Ratliff, Jr., Essential prime divisors and projectively equivalent ideals, J. Algebra, 109 (1987), 468-478. 
[6] - S. McAdam and L. J. Ratliff, Jr., Prime divisors and divisorial ideals, J. Pure Appl. Algebra, 59 (1989), 179-186.

[ 7 ] S. McAdam, Asymptotic Prime Divisors, Lecture Notes in Math., no. 1023, Springer, New York, 1983.

[ 8 ] — and L. J. Ratliff, Jr., On the asymptotic cograde of an ideal, J. Algebra, 87 (1984), 36-52.

[ 9 ] — and L. J. Ratliff, Jr., Essential sequences, J. Algebra, 95 (1985), 217-235.

[10] —, Quintessential primes and four results of Schenzel, J. Jure Appl. Algebra, 47 (1987), 283-298.

[11] M. Nagata, On the chain problem of prime ideals, Nagoya Math. J., 10 (1956), $51-64$.

[12] J. S. Okon, Asymptotic Prime Divisors and Filtrations, Ph. D. Dissertation, Univ. of California, Riverside, 1980.

[13] — and L. J. Ratliff, Jr., Filtrations, closure operations, and prime divisors, Math. Proc. Cambridge Philos. Soc., 104 (1988), 31-46.

[14] — and L. J. Ratliff, Jr., Filtrations, prime divisors, and Rees rings, Houston J. Math., 18 (1990), 387-405.

[15] — and L. J. Ratliff, Jr., Reductions of filtrations, Pacific J. Math., 144 (1990), $137-154$.

[16] L. J. Ratliff, Jr., Notes on essentially powers filtrations, Michigan Math. J., 26 (1979), 313-324.

[17] —- Asymptotic sequences, J. Algebra, 85 (1983), 337-360.

[18] — Note on analytic spread and asymptotic sequences, Math. Proc. Cambridge Philos. Soc., 93 (1983), pp. 49-55.

[19] —, On asymptotic prime divisors, Pacific J. Math., 111 (1984), 395-413.

[20] —- Asymptotic sequences and Rees rings, J. Algebra, 89 (1984), 65-87.

[21] - Five notes on asymptotic prime divisors, Math. Z., 190 (1985), 567-581.

[22] - Asymptotic prime divisors and integral extension rings, J. Algebra, 95 (1985), 409-431.

[23] —-, Essential sequences over an ideal and essential cograde, Math. Z., 188 (1985), 383-395.

[24] —_ Essential sequences and Rees rings, J. Algebra, 99 (1986), 337-354.

[25] - and D. E. Rush, Note on I-good filtrations and Noetherian Rees rings, Comm. Algebra, 16 (1988), 955-975.

[26] - Notes on projectively related ideas and residual division, J. Algebra, 130 (1990), 435-450.

[27] D. Rees, Rings associated with ideals and analytic spread, Math. Proc. Cambridge Philos. Soc., 89 (1981), 423-432.

[28] —- Asymptotic Properties of Ideals, Nagoya Lecture Notes, preprint.

[29] —-, Semi-Noether filtrations: I, J. London Math. Soc., 37 (1988), 43-62.

\section{J. S. Okon}

Department of Mathematics

California State University

San Bemardino, California 92407

L. J. Ratliff, Jr.

Department of Mathematics

University of California

Riverside, California 92521 